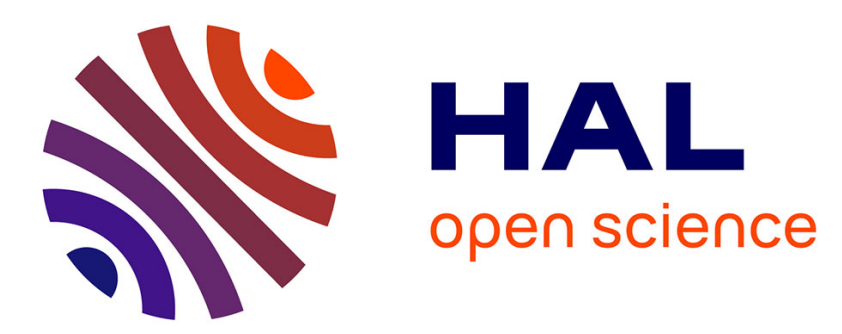

\title{
Coupled lattice Boltzmann method for numerical simulations of fully coupled heart and torso bidomain system in electrocardiology
}

Samuel Corre, Aziz Belmiloudi

\section{- To cite this version:}

Samuel Corre, Aziz Belmiloudi. Coupled lattice Boltzmann method for numerical simulations of fully coupled heart and torso bidomain system in electrocardiology. Journal of Coupled Systems and Multiscale Dynamics, 2016, 4 (3), pp.207-229. 10.1166/jcsmd.2016.1109 . hal-01477278

\section{HAL Id: hal-01477278 \\ https://hal.science/hal-01477278}

Submitted on 4 Apr 2017

HAL is a multi-disciplinary open access archive for the deposit and dissemination of scientific research documents, whether they are published or not. The documents may come from teaching and research institutions in France or abroad, or from public or private research centers.
L'archive ouverte pluridisciplinaire HAL, est destinée au dépôt et à la diffusion de documents scientifiques de niveau recherche, publiés ou non, émanant des établissements d'enseignement et de recherche français ou étrangers, des laboratoires publics ou privés. 


\title{
Coupled Lattice Boltzmann Method for Numerical Simulations of Fully Coupled Heart and Torso Bidomain System in Electrocardiology
}

\author{
S. Corre \\ INSA-IRMAR, Rennes, Samuel.Corre@insa-rennes.fr \\ A. Belmiloudi \\ UEB-IRMAR, Rennes, France Aziz.Belmiloudi@math.cnrs.fr
}

S. Corre \& A. Belmiloudi, Coupled Lattice Boltzmann Method for Numerical Simulations of Fully Coupled Heart and Torso Bidomain System in Electrocardiology, J. Coupled Systems and Multiscale Dynamics, Vol. 4, N 3, 207-229 (2016).

\begin{abstract}
In this work, a modified coupling Lattice Boltzmann Model (LBM) in simulation of cardiac electrophysiology is developed in order to capture the detailed activities of macro- to micro-scale transport processes. The propagation of electrical activity in the human heart through torso is mathematically modeled by bidomain type systems. As transmembrane potential evolves, we take into account domain anisotropical properties using intracellular and extracellular conductivity, such as in a pacemaker or an electrocardiogram, in both parallel and perpendicular directions to the fibers. The bidomain system represents multi-scale, stiff and strongly nonlinear coupled reaction-diffusion models that consist of a set of ordinary differential equations coupled with a set of partial differential equations. Due to dynamic and geometry complexity, numerical simulation and implementation of bidomain type systems are extremely challenging conceptual and computational problems but are very important in many real-life and biomedical applications. This paper suggests a modified LBM scheme, reliable, efficient, stable and easy to implement in the context of such bidomain systems. Numerical tests to confirm effectiveness and accuracy of our approach are provided and the propagation of electrophysiological waves in the heart is analyzed.

Keywords: Coupled Lattice Boltzmann, Heart-torso coupling, coupled nonlinear parabolic/elliptic PDEs, bidomain models, Ionic models, cardiac electrophysiology, .
\end{abstract}

\section{Introduction}

Computational cardiac electrophysiological modeling is now an important field in applied mathematics. Indeed, nowadays, heart and cardiovascular diseases are still the leading cause of death and disability all over the world. That is why we need to improve our knowledge about heart behavior, and more particularly about its electrical behavior. Consequently we want strong methods to compute electrical fluctuations in the torso region to prevent cardiac disorders (as arrhythmias), or to study interactions between brain and heart.

In this work, coupling Lattice Boltzmann Models (LBM) in simulation of cardiac electrophysiology are developed in order to capture the detailed activities of macro- to micro-scale transport processes. The propagation of electrical activity in the human heart is mathematically modeled by bidomain type systems in heart, derived from Ohm's law, 
and is coupled with a general Laplace equation in surrounding tissues across the torso. As heart is embedded in the torso, the dynamic of electrical potential in torso have to be treated in detail to obtain realistic behavior in myocardium (the muscle tissue of the heart) for several applications such as in a pacemaker or an electrocardiogram. The muscle tissue of the ventricles and atria are composed of fibers which is oblong like cylinders and formed by thick and thin protein filaments. Each fiber is connected to neighboring fibers with a step-like surface. Because of these connections, the electrical current passes from cell to cell and its direction is therefore determined by the orientation of the fibers. This makes the myocardial tissue anisotropic, and we use intracellular and extracellular conductivity, with two tensors respectively called $\mathcal{K}_{i c}$ and $\mathcal{K}_{e c}$, in both parallel and perpendicular directions to those fibers to taking into account the anisotropy. So, even if this tissue has a granular nature and is composed of multiple individual cells, we can consider this tissue as continuous anisotropic domain which can be likened to a functional syncytium. This biophysical model of electrical cardiac activity links electrophysiological cell models, at small scales, and myocardial tissue mechanics, metabolism and blood flow at large scales. Indeed, myocardium fibers have a higher conductivity than normal muscle fibers. That is because the heart cells are surrounded by a plasma membrane which induces a different chemical and electrical intracellular and extracellular behavior. There is notably a higher intracellular potassium concentration than in the extracellular fluid. This difference induces an outflow of potassium which can be traduced by a difference of distribution charge, called the transmembrane potential $\rho$. External electrical stimulus control ion channels gates in the membrane, and intracellular and extracellular ionical concentration changes while the transmembrane potential leaves its resting state (see e.g. [14], [24] and the references therein).

So, the bidomain system represents multi-scale, stiff and strongly nonlinear coupled reaction-diffusion models (governing the dynamics at cellular and tissue levels) that consists of a set of ordinary differential equations coupled with a set of partial differential equations in myocard to compute extracellular potential $\rho_{e}$, transmembrane potential $\rho$ and ionic variable $U$, which are linked to the Laplace equation in torso to compute torso potential $\rho_{T}$ with Robin type boundary conditions between torso and extra-cellular potentials. During last years a lot of studies about bidomain models have led to results about well-posedness, existence and uniqueness of solutions (see e.g., [3] , [9] and the references therein), and several numerical methods based on methods as finite difference method or finite element method are used to solve these models (see e.g., [31] and the references therein).

However, there exist several models to simulate electrical behavior in electrophysiology based on Hodgkin-Huxley equations to approximate the electrical characteristics of excitable cells such as neurons and cardiac myocytes. In order to have a simple and realistic model, we choose 2-variable phenomenological Mitchell-Schaeffer (MS) model [26] to capture ionic phenomena. This model derived from Fenton-Karma 3-variable model [19] is interesting because of its simplicity. In the same time, it has already shows its accuracy and its realism. Due to dynamic and geometry complexity, numerical simulation and implementation of bidomain type systems are extremely challenging conceptual and computational problems but are very important in many real-life and biomedical applications. This work presents a modified coupled LBM scheme, reliable, efficient, stable and easy to implement in the context of such bidomain systems with Neumann type boundary conditions in complex geometry boundaries. LBM was originated from Boltzmann's kinetic theory of gases $(70 \mathrm{~s})$, and attracts more and more attentions for simulating complex fluid flows since 90s. More recently, LBM has been extended successfully to simulate different types of parabolic reaction-diffusion equation as Keller-Segel chemotaxis model [35] and monodomain model in cardiac electrophysiology [11], or Poisson equation [12]. The concept of LBM, which is efficient in terms of parallelization, is based on Boltzmann equations which describe the evolution of particles in kinetic theory. LBM has two main phases: one, local, which models the collision between particles and the second, along each direction of interpolation, which models the transport phase. We demonstrate that solving Lattice Boltzmann Equations (LBE) is equivalent to solving governing bidomain system 
with Chapman-Enskog perturbation expansion [13]. This ansatz leads us to decompose the particular distributions $f$ around equilibrium state in different scales of perturbation with introduction of dimensionless Knudsen number $\varepsilon$ which is a ratio between the particular mean free path length to a representative physical length scale. So, for every distribution function we can rewrite distribution function $f$ as $f=f^{e q}+\varepsilon f^{(1)}+\varepsilon^{2} f^{(2)}+\ldots$. Then, the main point of Chapman-Enskog expansion is to match LBE with Taylor series with including the decomposition in order to recompose the governing bidomain system. Finally, we propose two applications in simplified square domains for heart and torso. On the one hand, numerical results demonstrate the effectiveness and accuracy of our approach using general methods for bidomain type systems with full known data and chosen analytical solution. On the other one, we treat the model with realistic data to show good agreement with numerical results and potential behavior reported in the literature (see e.g., [7], [29]).

The paper is structured as follows: first, in Section 2 we introduce the full coupled heart and torso bidomain system and the MS ionic model. Then, in Section 3 after some generalities about LBM, we develop our modified coupled LBM for numerical simulations of the considered model and investigate its asymptotic behavior as $\epsilon$ goes to zero. The validity of this method is demonstrated in Section 4 by comparing the numerical solution to the known exact solution, and convergence of solution is established. Finally, the propagation of electrophysiological waves in the heart is studied.

\section{The bidomain Model}

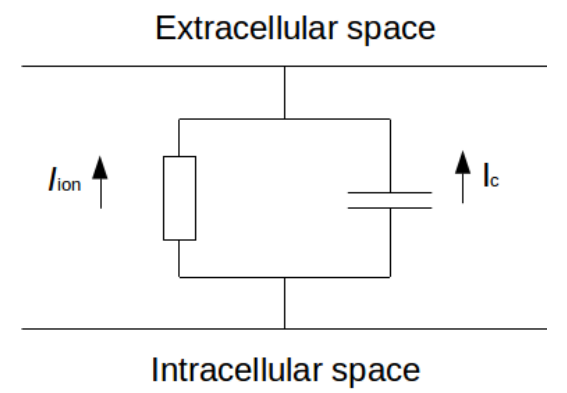

Figure 1: Modeling of the membrane as resistor and capacitor coupled in parallel.

The full coupled heart and torso bidomain model of cardiac tissue is expressed mathematically by the following time dependent system of coupled partial differential equations governing the electrical potentials in the physical region $\Omega=\Omega_{H} \cup \Omega_{T}$, which is an open, bounded, and connected subset of $\mathbf{R}^{d}, d \leq 3$ and during a time interval $(0, T)$. The two spaces $\Omega_{H}$ and $\Omega_{T}$ are the regions occupied by the excitable myocardium tissue (the heart muscle) and the thorax, respectively.

First, we will introduce the bidomain model in myocard $\Omega_{H}$. Propagation of electrical potential is modeled by:

$$
\operatorname{div}\left(\mathcal{K}_{i c} \nabla \rho_{i}\right)=I_{m}-\kappa f_{i s}, \quad \operatorname{div}\left(\mathcal{K}_{e c} \nabla \rho_{e}\right)=-I_{m}-\kappa f_{e s},
$$

where $\rho_{i}$ and $\rho_{e}$ are the intracellular and extracellular potentials, respectively; $\mathcal{K}_{i c}$ and $\mathcal{K}_{e c}$ are the conductivity tensors describing the anisotropic intracellular and extracellular conductive media; $f_{i s}(x, t)$ and $f_{e s}(x, t)$ are the respective externally applied current sources after they passed through the thorax. The transmembrane current density is described by $I_{m}$ and is given by the following expression:

$$
I_{m}=\kappa\left(c_{m} \frac{\partial \rho}{\partial t}+\mathcal{I}_{i o n}\right)
$$


where $\rho$ is the transmembrane potential, which is defined as $\rho=\rho_{i}-\rho_{e}, \kappa$ is the ratio of the membrane surface area to the volume occupied by the tissue, $c_{m}$ term is the transmembrane capacitance time unit area. We can assimilate this membrane to a simple electrical circuit with a resistor associate to the ionic current $\left(\mathcal{I}_{i o n}\right)$ and a capacitor associate to the capacitive current $\left(I_{c}=c_{m} \frac{\partial \rho}{\partial t}\right)$ in parallel (Figure 1). The tissue is assumed to be passive, so the capacitance $c_{m}$ can be assumed to be not a function of the state variables. The nonlinear operator $\mathcal{I}_{i o n}(x, t ; \rho, U)$ describes the sum of transmembrane ionic currents across the cell membrane with $U$ the electrophysiological ionic state variable (which describes e.g., the dynamics of ion-channel and ion concentrations in different cellular compartments). These variables satisfy the following ODE (with $H_{\text {ion }}$ a nonlinear operator)

$$
\frac{\partial U}{\partial t}=H_{i o n}(x, t ; \rho, U)
$$

From (1), (2) and (3), the bidomain model can be formulated in terms of the state variables $\rho, \rho_{e}$ and $U$ as follows (in $\mathcal{Q}_{H}=\Omega_{H} \times(0, T)$ )

$$
\begin{aligned}
& \kappa\left(c_{m} \frac{\partial \rho}{\partial t}+\mathcal{I}_{i o n}(. ; \rho, U)\right)-\operatorname{div}\left(\mathcal{K}_{i c} \nabla \rho\right)=\operatorname{div}\left(\mathcal{K}_{i c} \nabla \rho_{e}\right)+\kappa f_{i s}, \\
& -\operatorname{div}\left(\left(\mathcal{K}_{e c}+\mathcal{K}_{i c}\right) \nabla \rho_{e}\right)=\operatorname{div}\left(\mathcal{K}_{i c} \nabla \rho\right)+\kappa\left(f_{e s}+f_{i s}\right), \\
& \frac{\partial U}{\partial t}=H_{i o n}(. ; \rho, U) .
\end{aligned}
$$

The operators $\mathcal{I}_{i o n}$ and $H_{i o n}$ which describe electrophysiological behavior depends on the considered cell ionic model. We can choose physiological ionic models (e.g Djabella and Sorine [16] or Luo and Rudy [25]), phenomenological models (e.g Fenton-Karma [19], Fitzhugh-Nagumo [27], or Mitchell-Schaeffer [26]). In our case, we choose the phenomenological two-variable classical model proposed by Mitchell and Schaeffer as:

$$
\begin{aligned}
& \mathcal{I}_{\text {ion }}(\mathbf{x}, t ; \rho, U)=I_{1}(\mathbf{x}, t ; \rho)+I_{2}(\mathbf{x}, t ; \rho) U, \\
& H_{\text {ion }}(\mathbf{x}, t ; \rho, U)=\left\{\begin{array}{l}
\lambda_{1}(\mathbf{x}) U+H_{0}(\mathbf{x}, t) \text { if } \rho \leq \rho_{\text {gate }}, \\
\lambda_{2}(\mathbf{x}) U \text { if } \rho>\rho_{\text {gate }},
\end{array}\right.
\end{aligned}
$$

where operators $I_{1}, I_{2}$, functions $\lambda_{1}, \lambda_{2}$ and $H_{0}$ depend of given parameters and where $\rho_{\text {gate }}$ is a given activation threshold.

Then, under the assumption that the heart is isolated from its surrounding (e.g. noncoupled with the thorax), we will search $\rho_{T}$ which is electrical potential in thorax with solving propagation equation (in $\mathcal{Q}_{T}=\Omega_{T} \times(0, T)$ ):

$$
-\operatorname{div}\left(\mathcal{K}_{T} \nabla \rho_{T}\right)=f_{T},
$$

where $\mathcal{K}_{T}$ is the conductivity tensor describing the anisotropic conductivity in thorax, and $f_{T}$ is the applied current source.

To close the system, we impose the following boundary conditions on $\Sigma=\partial \Omega_{H} \times(0, T)$

$$
\left(\mathcal{K}_{i c} \nabla\left(\rho+\rho_{e}\right)\right) \cdot \mathbf{n}=\zeta_{i}, \quad\left(\mathcal{K}_{e c} \nabla \rho_{e}\right) \cdot \mathbf{n}=\zeta_{e},
$$

and on $\Sigma_{T}=\Gamma_{T} \times(0, T)$ where $\Gamma_{T}=\partial \Omega_{T} \backslash \partial \Omega_{H}$ :

$$
\left(\mathcal{K}_{T} \nabla \rho_{T}\right) \cdot \mathbf{n}=0
$$

where $\mathbf{n}$ being the outward normal to $\Gamma=\partial \Omega_{H}$ and $\zeta_{i}$ and $\zeta_{e}$ are the intra- and extra-cellular currents per unit area applied across the boundary, and the following initial conditions (in $\Omega_{H}$ )

$$
\rho(t=0)=\rho_{0}, \quad U(t=0)=U_{0} .
$$

Such problems have compatibility conditions determining whether there are any solutions to the PDEs. This is easily found by integrating the second equation of (4) over the domain and using the divergence theorem with the boundary conditions (7) (a.e. in $(0$, $\mathrm{T})$ ). Then (for compatibility reasons), we require the following condition

$$
\int_{\Gamma}\left(\zeta_{i}+\zeta_{e}\right) d \Gamma+\kappa \int_{\Omega_{H}}\left(f_{e s}+f_{i s}\right) d x=0 .
$$


Moreover, the function $\rho_{e}$ is defined within a class of equivalence, regardless of a timedependent function. This function can be fixed, for example by setting the following condition (a.e. in $(0, T)$ )

$$
\int_{\Omega_{H}} \rho_{e} d x=0
$$

Under some hypotheses for the data and parameters of the system and some regularity of operators $\mathcal{I}_{i o n}$ and $H_{i o n}$, system (4) with (7)-(9) and under the conditions (10)-(11) is a well-posed problem (for more details see $[3,9]$ ).

We respect the following boundary conditions on $\Sigma$ :

$$
\left(\mathcal{K}_{i c} \nabla \rho+\left(\mathcal{K}_{i c}+\mathcal{K}_{e c}\right) \nabla \rho_{e}\right) \cdot \mathbf{n}=\left(\mathcal{K}_{T} \nabla\left(\rho_{T}\right)\right) \cdot \mathbf{n},
$$

which induce the following boundary condition:

$$
\left(\mathcal{K}_{T} \nabla \rho_{T}\right) \cdot \mathbf{n}=\zeta_{e}+\zeta_{i} \quad \text { on } \Sigma .
$$

Remark 2.1 .

In order to guarantee the well-posedness of system (4)-(9) (under some hypotheses for data and some regularity of the nonlinear operators), we can use the following regularized version of ionic operators $H_{\text {ion }}$, given by (5), (see e.g., [8])

$$
\begin{aligned}
H_{r}(\mathbf{x}, t ; \rho, U)= & \left(-\lambda_{2}(\mathbf{x})+\left(\lambda_{2}(\mathbf{x})-\lambda_{1}(\mathbf{x})\right) h_{\infty}(\rho)\right)\left(U(\mathbf{x}, t)-h_{\infty}(\rho)\right) \\
& -\left(H_{0}(\mathbf{x}, t)+\lambda_{1}(\mathbf{x})\right) h_{\infty}(\rho),
\end{aligned}
$$

where $h_{\infty}(\rho)$ is given by

$$
h_{\infty}(\rho)=\frac{1}{2}\left(1-\tanh \left(\frac{\rho-\rho_{\text {gate }}}{r_{g}}\right)\right),
$$

with $r_{g}$ a positive parameter. We can deduce that

$$
\lim _{r_{g} \rightarrow 0} h_{\infty}(\rho)=\left\{\begin{array}{l}
1 \text { if } \rho<\rho_{\text {gate }} \\
0 \text { if } \rho>\rho_{\text {gate }}
\end{array}\right.
$$

To illustrate the convergence of $H_{r}$ to $H_{\text {ion }}$, we consider, e.g., the following functions

$$
\begin{aligned}
& \lambda_{1}(\mathbf{x})=\frac{1}{\mathcal{T}_{\text {open }}}, \lambda_{2}(\mathbf{x})=\frac{1}{\mathcal{T}_{\text {close }}}, \\
& H_{0}(\mathbf{x}, t)=-\frac{1}{\mathcal{T}_{\text {open }}\left(\rho_{\text {max }}-\rho_{\text {min }}\right)^{2}} .
\end{aligned}
$$

with $\rho_{\text {gate }}=-67 \mathrm{mV}, \mathcal{T}_{\text {open }}=100 \mathrm{~ms}, \mathcal{T}_{\text {close }}=120 \mathrm{~ms}$, and we choose $U(\mathbf{x}, t)=10^{-3}$. As Figure 2 shows for different parameters $r_{g}, \lim _{r_{g} \rightarrow 0} H_{r}(\rho, U)=H_{\text {ion }}(\rho, U)$.

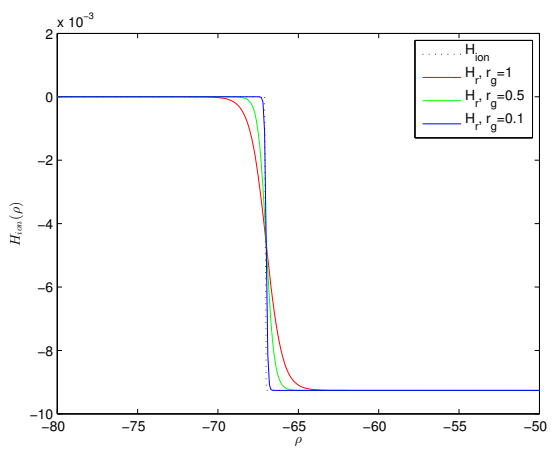

Figure 2: Operator $H_{\text {ion }}$ and regularized operator $H_{r}$ for $r_{g}=1, r_{g}=0.5, r_{g}=0.1$. 


\section{$3 \quad$ Numerical method and algorithm}

In this section, a numerical method is presented for the bidomain system (4) in two space dimensions. For this, we introduce a coupled modified LBM for solving the coupled system of nonlinear parabolic and elliptic equations (i.e. the first and second equations of (4)). Then we treat the ODE satisfied by ionic state by applying Gronwall Lemma to obtain an integral formulation, and by using a quadrature rule to approximate the obtained integral.

In the sequel, without loss of generality, we assume $c_{m}=1$ and $\kappa=1$. Moreover we assume $\mathcal{K}_{i c}=K_{i c} \mathbf{I}_{d}, \mathcal{K}_{e c}=K_{e c} \mathbf{I}_{d}, \mathcal{K}_{T}=K_{T} \mathbf{I}_{d}$, with $\mathbf{I}_{d}$ identity matrix and $K_{i c}, K_{e c}$, $K_{T}$ constants.

\subsection{LBM for coupled parabolic and elliptic equations}

In this first part, we develop and describe the modified LBM to solve the following system (which corresponds to two first parts of (4) and elliptic equation in thorax (6))

$$
\begin{aligned}
& \frac{\partial \rho}{\partial t}-\operatorname{div}\left(K_{i c} \nabla\left(\rho+\rho_{e}\right)\right)=F\left(. ; \rho, \rho_{e}\right), \text { in } \mathcal{Q}_{H} \\
& -\operatorname{div}\left(K_{i c} \nabla \rho+\left(K_{i c}+K_{e c}\right) \nabla \rho_{e}\right)=G\left(. ; \rho, \rho_{e}\right), \text { in } \mathcal{Q}_{H} \\
& -\operatorname{div}\left(K_{T} \nabla \rho_{T}\right)=H\left(. ; \rho_{T}\right), \text { in } \mathcal{Q}_{T}
\end{aligned}
$$

where $F, G$ and $H$ are given nonlinear operators.

Before introducing the coupled LBM for system (14), it is important to explain the main steps of this method and to give some definitions about discretization and operators in case of a general reaction-diffusion equation.

\subsubsection{LBM for general reaction-diffusion equations}

Let us introduce the LBM for solving the following reaction-diffusion equation (with the macroscopic variable $\Theta$ )

$$
\frac{\partial \Theta(\mathbf{x}, t)}{\partial t}-\operatorname{div}(K(\mathbf{x}, t) \nabla \Theta(\mathbf{x}, t))=\Phi(\mathbf{x}, t ; \Theta),
$$

where $\Phi$ is a non linear operator and $K$ is a diffusion coefficient. The evolution equation of the LBM for (15) is given by the following continuous Boltzmann equation [6]:

$$
\frac{\partial \phi(\mathbf{x}, t ; \mathbf{e})}{\partial t}+\mathbf{e} \cdot \nabla \phi(\mathbf{x}, t ; \mathbf{e})=\mathcal{J}(\mathbf{x}, t ; \mathbf{e})
$$

with

$$
\Theta(\mathbf{x}, t)=\int \phi(\mathbf{x}, t ; \mathbf{e}) d \mathbf{e}
$$

where $\mathcal{J}(\mathbf{x}, t ; \mathbf{e})=Q_{c o l}(\phi(\mathbf{x}, t ; \mathbf{e}))+P(\mathbf{x}, t ; \mathbf{e}), \phi(\mathbf{x}, t ; \mathbf{e})$ is the distribution function of the single particle moving with velocity $\mathbf{e}$ at position $\mathbf{x}$ and time $t, P$ is the distribution type function of particle of macroscopic external force $\Phi$ moving with velocity e and $Q_{c o l}$ is the Bhatnagar-Gross-Krook (BGK) collision operator defined by (see [5]):

$$
Q_{c o l}(\phi(\mathbf{x}, t ; \mathbf{e}))=-\frac{1}{\tau(\mathbf{x}, t)}\left(\phi(\mathbf{x}, t ; \mathbf{e})-\phi^{e q}(\mathbf{x}, t ; \mathbf{e})\right),
$$

where $\phi^{e q}$ is the Maxwell-Boltzmann equilibrium distribution function and $\tau$ is the dimensionless relaxation time.

LBM leads us to approximate (16) to recover reaction-diffusion equation (15) with Chapman-Enskog expansion. In the method of Chapman-Enskog the distribution function

$\phi$ is expanded as : $\phi=\phi^{(0)}+\epsilon \phi^{(1)}+\epsilon^{2} \phi^{(2)}+\ldots .=\sum_{k=0}^{\infty} \epsilon^{k} \phi^{(k)}$, which can be regarded as a power series in a small variable $\epsilon$ or an expression that keeps track of the relative orders of magnitude of the different terms through the scaling parameter $\epsilon$ (the so-called Kundsen number). The distribution functions $\phi^{(0)}, \phi^{(1)}$ and $\phi^{(2)}$ represent the zero, first and second approximation to the distribution function $\phi$, and so on. 


\section{Remark 3.1 .}

It is important to note that $\phi^{e q}$ is an exact solution of the Chapman-Enskog's zero-order approximation of the Boltzmann equation (see [22]).

The numerical solution of Boltzmann equation (16) requires to discretize the configuration spaces, velocities and time. For that, we discretize $\mathcal{Q}=\Omega \times(0, T)$ in time and space. Then we consider a regular lattice $\mathcal{L}$ with typical mesh size $\Delta x$ and time step size $\Delta t$, and we define streaming lattice speed $c=\Delta x / \Delta t$ and lattice sound speed $C_{s}$ by $3 C_{s}^{2}=c^{2}$. We note $\left(\mathbf{e}_{i}\right)_{i=0, N-1}$ the discrete set of $N$ admissible particle velocities (the magnitude of each $\mathbf{e}_{i}$ depends on the speed $c$ ) and we assume that for each node $\mathbf{x}$ of lattice $\mathcal{L}$, and each $\mathbf{e}_{i}$, the point $\mathbf{x}+\mathbf{e}_{i} \Delta t$ is also a node of the lattice $\mathcal{L}$. For the latter, we use the so-called $D 2 Q N$ LBM scheme (i.e., 2-dimensional and $N$ velocity vectors).

\section{Remark 3.2 .}

Since the velocities $\mathbf{e}_{i}$ are constant vectors in space-time variables, then, for sufficiently regular scalar function $\Xi$, we have

$$
\mathbf{e}_{i} \cdot \nabla \Xi=\operatorname{div}\left(\mathbf{e}_{i} \Xi\right) .
$$

First, we consider the finite discrete-velocity system of the Boltzmann equation with the finite discrete velocity $\mathbf{e}_{i}$, for $i=0, N-1$ (by discretizing the velocity space by the discrete set of microscopic velocities)

$$
\frac{\partial \phi_{i}(\mathbf{x}, t)}{\partial t}+\mathbf{e}_{i} \cdot \nabla \phi_{i}(\mathbf{x}, t)=\mathcal{J}_{i}(\mathbf{x}, t)
$$

where, for each particle on the lattice, we associate discrete functions, for $i=0, N-1$, $\phi_{i}(\mathbf{x}, t)=\phi\left(\mathbf{x}, t ; \mathbf{e}_{i}\right), \phi_{i}^{e q}(\mathbf{x}, t)=\phi^{e q}\left(\mathbf{x}, t ; \mathbf{e}_{i}\right)$ and $\mathcal{J}_{i}(\mathbf{x}, t)=\mathcal{J}\left(\mathbf{x}, t ; \mathbf{e}_{i}\right)$, and the discrete operator $\Phi_{i}$ of $\Phi$, which describes the probability of streaming in one particular direction.

The aim of the so-called LBM scheme is to compute the distribution function of particle $\left(\phi_{i}(\mathbf{x}, t)\right)_{i=1, N}$ for $\mathbf{x} \in \mathcal{L}$ and discrete values of time $t$ by solving the discretization of the Boltzmann's equation (20) in two steps (for each time): collision and streaming processes. Then, by using a second order time integration scheme to approximate (20) (by limiting physical space to a lattice and velocity space to the discrete set of microscopic velocities), we can obtain

$$
\begin{aligned}
\phi_{i}\left(\mathbf{x}+\mathbf{e}_{i} \Delta t, t+\Delta t\right)= & \phi_{i}(\mathbf{x}, t)-\frac{1}{\tau(\mathbf{x}, t)}\left(\phi_{i}(\mathbf{x}, t)-\phi_{i}^{e q}(\mathbf{x}, t)\right) \\
& +\Delta t \Phi_{i}(\mathbf{x}, t)+\frac{\Delta t^{2}}{2}\left(\frac{\partial}{\partial t}+\mathbf{e}_{i} \cdot \nabla\right) \Phi_{i}(\mathbf{x}, t),
\end{aligned}
$$

which can be decomposed into the two key sub-steps i.e. collision and streaming. The collision process is local in space and is given for each node $\mathbf{x}$ by:

$$
\begin{aligned}
\phi_{i}^{c o l}(\mathbf{x}, t) & =\phi_{i}(\mathbf{x}, t)-\frac{1}{\tau(\mathbf{x}, t)}\left(\phi_{i}(\mathbf{x}, t)-\phi_{i}^{e q}(\mathbf{x}, t)\right) \\
& +\Delta t \Phi_{i}(\mathbf{x}, t)+\frac{\Delta t^{2}}{2}\left(\frac{\partial}{\partial t}+\mathbf{e}_{i} \cdot \nabla\right) \Phi_{i}(\mathbf{x}, t)
\end{aligned}
$$

Then we can formally summarize the streaming step as a translation in post-collision distribution function fields. For each node, the post-collision distribution function $\phi_{i}^{c o l}(\mathbf{x}, t)$ will replace post-collision distribution function of the neighbor node $\phi_{i}^{c o l}\left(\mathbf{x}+\mathbf{e}_{i} \Delta t, t\right)$ according to velocity $\mathbf{e}_{i}$ (Fig. 4 ). So, streaming process is given for each velocity $\mathbf{e}_{i}$ and in a step time $\Delta t$ by:

$$
\phi_{i}\left(\mathbf{x}+\mathbf{e}_{i} \Delta t, t+\Delta t\right)=\phi_{i}^{c o l}(\mathbf{x}, t) \quad \text { (the propagation of Lattice Boltzmann scheme). }
$$

Finally, we recover governing macroscopic variable $\Theta$ by a summation of microscopic particle distribution function $\phi_{i}, i=1, N$ as follows (which is a discrete approximation 
of the integral given in (17))

$$
\sum_{i=0}^{N} \phi_{i}(\mathbf{x}, t)=\Theta(\mathbf{x}, t), \quad \text { for } \mathbf{x} \in \mathcal{L} \text { and discrete values of time } t .
$$

In this paper, we consider the popular two-dimensional $D 2 Q 9$ lattice, which involves 9 velocity vectors (i.e. $N=9$ ). The microscopic velocities $\mathbf{e}_{i}$, for $i=0,8$ in $D 2 Q 9$ are given as (see Figure 3)

$$
\begin{gathered}
\mathbf{e}_{0}=\left(\begin{array}{l}
0 \\
0
\end{array}\right), \\
\mathbf{e}_{i}=c\left(\begin{array}{c}
\cos \left((i-1) \frac{\pi}{2}\right) \\
\sin \left((i-1) \frac{\pi}{2}\right)
\end{array}\right), \text { for } i=1,2,3,4, \\
\left.\mathbf{e}_{i}=\sqrt{2} c\left(\begin{array}{c}
\cos \left(\left(i-\frac{9}{2}\right) \frac{\pi}{2}\right) \\
\sin \left(\left(i-\frac{9}{2}\right) \frac{\pi}{2}\right.
\end{array}\right)\right), \text { for } i=5,6,7,8 .
\end{gathered}
$$

According to the expression of the microscopic velocities $\mathbf{e}_{i}$, we prove easily that

$$
\sum_{i=0}^{8} \mathbf{e}_{i}=\sum_{i=0}^{8} w_{i} \mathbf{e}_{i}=\left(\begin{array}{l}
0 \\
0
\end{array}\right)
$$

and

$$
\sum_{i=0}^{8} w_{i} \mathbf{e}_{i} \mathbf{e}_{i}=C_{s}^{2} \mathbf{I}_{d}
$$

where the weight vector $\mathbf{w}=\left(w_{0}, \ldots, w_{8}\right)$ is defined by

$$
\begin{aligned}
& \sum_{i=0}^{8} w_{i}=1, \quad w_{0}=4 / 9 \\
& w_{1}=w_{2}=w_{3}=w_{4}=1 / 9 \text { and } w_{5}=w_{6}=w_{7}=w_{8}=1 / 36
\end{aligned}
$$

\section{Remark 3.3 .}

With using D2Q9 model, we define a 9-speed square lattice with square elements. It is also possible to use D2Q7 model to obtain a 7-speed hexagonal lattice with triangular elements or to define other types of quadrature if it is needed. In all cases, we have to care about geometric properties. Indeed, respect summations (25) and (26) is necessary to obtain a convergent method.
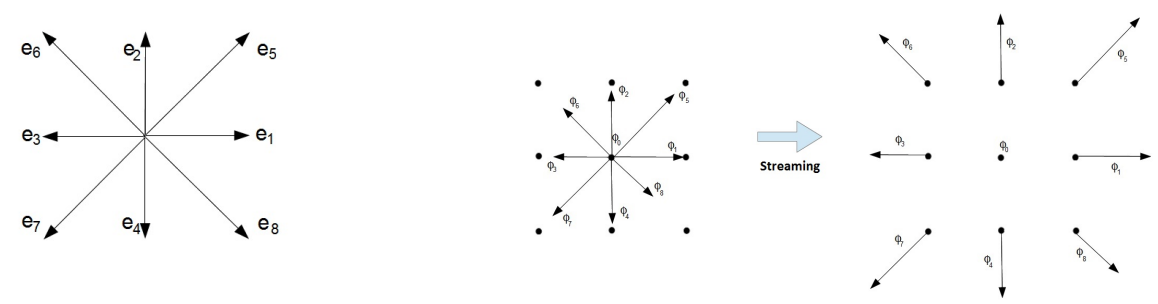

Figure 3: Particle velocities for D2Q9 LBM

Figure 4: Streaming process of a lattice node

\section{Remark 3.4 .}

During streaming and collision processes, in order to satisfy boundary conditions, the boundary nodes need special treatments on distribution functions, which are essential to stability and accuracy of the method. We use extrapolation methods [20] and modified bounce-back methods [37], [23], [36]. 


\subsubsection{LBM for coupled system}

In this part, we detail the modified coupled LBM presented in [15], adapted from [35] with introducing LBM for elliptic equations proposed in [12]. We begin with the introduction of three Lattice Boltzmann type equations, which are associated to the three equations of the coupled system of parabolic and elliptic equations (14), respectively. Then, we define discrete equilibrium functions, source terms, nonlinear operators and relaxation time associated to each equation as in previous section. So, we associate the repartition functions of particle moving with velocity $\mathbf{e}_{i}$, with $i=0, \ldots, 8, f_{i}, g_{i}$ and $h_{i}$ for $\rho, \rho_{e}$ and $\rho_{T}$, respectively.

The discrete LBM equation for the first equation of (14), which is parabolic, can be given by (at node $\mathbf{x}$ and time variable $t$ )

$$
\begin{aligned}
f_{i}\left(\mathbf{x}+\mathbf{e}_{i} \Delta t, t+\Delta t\right)= & f_{i}(\mathbf{x}, t)-\frac{1}{\tau_{1}(\mathbf{x}, t)}\left(f_{i}(\mathbf{x}, t)-f_{i}^{e q}(\mathbf{x}, t)\right) \\
& +\Delta t S_{i}^{1}\left(\mathbf{x}, t ; \nabla \rho, \nabla \rho_{e}\right) \\
& +\Delta t F_{i}(\mathbf{x}, t)+\frac{\Delta t^{2}}{2}\left(\frac{\partial}{\partial t}+\mathbf{e}_{i} \cdot \nabla\right) F_{i}(\mathbf{x}, t),
\end{aligned}
$$

where $\tau_{1}(\mathbf{x}, t)$ is the dimensionless relaxation factor, $F_{i}(\mathbf{x}, t)$ is the discrete source term associated to operator $F\left(\mathbf{x}, t ; \rho, \rho_{e}\right), S_{i}^{1}\left(\mathbf{x}, t ; \nabla \rho, \nabla \rho_{e}\right)$ is a link function (between the first and second equations of (14)) related to gradients $\nabla \rho$ and $\nabla \rho_{e}$.

For the two elliptic equations of (14), for a fixed time $t$, the discrete pseudo-timestepping LBM equations can be given (at node $\mathbf{x}$ and pseudo time variables $r$ and $s$ )

$$
\begin{aligned}
g_{i}\left(\mathbf{x}+\mathbf{e}_{i} \Delta r, r+\Delta r ; t\right)= & g_{i}(\mathbf{x}, r ; t)-\frac{1}{\tau_{2}(\mathbf{x}, t)}\left(g_{i}(\mathbf{x}, r ; t)-g_{i}^{e q}(\mathbf{x}, r ; t)\right) \\
& +\Delta r S_{i}^{2}\left(\mathbf{x}, r ; t, \nabla \rho, \nabla \tilde{\rho}_{e}\right) \\
& +\Delta r G_{i}(\mathbf{x}, r ; t)+\frac{\Delta r^{2}}{2}\left(\frac{\partial}{\partial r}+\mathbf{e}_{i} \cdot \nabla\right) G_{i}(\mathbf{x}, r ; t), \\
h_{i}\left(\mathbf{x}+\mathbf{e}_{i} \Delta s, s+\Delta s ; t\right)= & h_{i}(\mathbf{x}, s ; t)-\frac{1}{\tau_{3}(\mathbf{x}, t)}\left(h_{i}(\mathbf{x}, t)-h_{i}^{e q}(\mathbf{x}, s ; t)\right) \\
& +\Delta s H_{i}(\mathbf{x}, s ; t)+\frac{\Delta s^{2}}{2}\left(\frac{\partial}{\partial s}+\mathbf{e}_{i} \cdot \nabla\right) H_{i}(\mathbf{x}, s ; t),
\end{aligned}
$$

where $\Delta r$ and $\Delta s$ are given time-steps, $\tau_{2}(\mathbf{x}, t)$ and $\tau_{3}(\mathbf{x}, t)$ are the dimensionless relaxation factors, $g_{i}(\mathbf{x}, r ; t)$ and $h_{i}(\mathbf{x}, s ; t)$ are the distribution functions of particle moving with velocity $\mathbf{e}_{i}$ at node $\mathbf{x}$ and times $r$ and $s, G_{i}(\underset{\mathbf{x}}{\tilde{x}}, r ; t)$ and $H_{i}(\mathbf{x}, s ; t)$ are the discrete source terms associated respectively to operators $\tilde{G}\left(\mathbf{x}, r ; t, \rho, \tilde{\rho}_{e}\right)$ and $\tilde{H}\left(\mathbf{x}, s ; t, \tilde{\rho}_{T}\right)$. The term $S_{i}^{2}\left(\mathbf{x}, r ; t, \nabla \rho, \nabla \tilde{\rho}_{e}\right)$ is the second link function (between the first and second equations of (14)) related to gradients $\nabla \rho$ and $\nabla \tilde{\rho}_{e}$.

Then, after each time step, we obtain new particular repartitions which lead us to recover governing macroscopic potentials $\rho, \tilde{\rho}_{e}$ and $\tilde{\rho}_{T}$ with following summations:

$$
\begin{array}{r}
\sum_{i=0}^{8} f_{i}(\mathbf{x}, t)=\rho(\mathbf{x}, t), \\
\frac{1}{1-w_{0}} \sum_{i=0}^{8} g_{i}(\mathbf{x}, r ; t)=\tilde{\rho}_{e}(\mathbf{x}, r ; t), \\
\frac{1}{1-w_{0}} \sum_{i=0}^{8} h_{i}(\mathbf{x}, s ; t)=\tilde{\rho}_{T}(\mathbf{x}, s ; t) .
\end{array}
$$

The two elliptic equations of system (14) associated to the pseudo time variables $r$ and $s$ induce subroutines in order to reach the following convergence:

$$
\lim _{r \rightarrow \infty} \tilde{\rho}_{e}(\mathbf{x}, r ; t)=\rho_{e}(\mathbf{x}, t)
$$




$$
\begin{aligned}
\lim _{s \rightarrow \infty} \tilde{\rho}_{T}(\mathbf{x}, s ; t) & =\rho_{T}(\mathbf{x}, t), \\
\lim _{r \rightarrow \infty} \tilde{G}\left(\mathbf{x}, r ; t, \rho, \tilde{\rho}_{e}\right) & =G\left(\mathbf{x}, t ; \rho, \rho_{e}\right)
\end{aligned}
$$

and

$$
\lim _{s \rightarrow \infty} \tilde{H}\left(\mathbf{x}, s ; t, \tilde{\rho}_{T}\right)=H\left(\mathbf{x}, t ; \rho_{T}\right) .
$$

Now we define equilibrium functions, source terms functions, linked functions and the three dimensionless factors $\tau_{1}, \tau_{2}, \tau_{3}$. The local equilibrium distribution functions are given by $(\forall i=0, \ldots, 8)$

$$
\begin{aligned}
f_{i}^{e q}(\mathbf{x}, t) & =w_{i} \rho(\mathbf{x}, t), \\
g_{i}^{e q}(\mathbf{x}, r ; t) & =\left\{\begin{array}{l}
w_{i} \tilde{\rho}_{e}(\mathbf{x}, r ; t) \text { for } i \neq 0, \\
\left(w_{0}-1\right) \tilde{\rho}_{e}(\mathbf{x}, r ; t) \text { for } i=0
\end{array}\right. \\
h_{i}^{e q}(\mathbf{x}, s ; t) & =\left\{\begin{array}{l}
w_{i} \tilde{\rho}_{T}(\mathbf{x}, s ; t) \text { for } i \neq 0, \\
\left(w_{0}-1\right) \tilde{\rho}_{T}(\mathbf{x}, s ; t) \text { for } i=0 .
\end{array}\right.
\end{aligned}
$$

According to (25) and (26), we can prove easily that

$$
\begin{gathered}
\sum_{i=0}^{8} f_{i}^{e q}(\mathbf{x}, t)=\rho(\mathbf{x}, t), \\
\sum_{i=0}^{8} \mathbf{e}_{i} f_{i}^{e q}(\mathbf{x}, t)=\left(\begin{array}{c}
0 \\
0
\end{array}\right), \\
\sum_{i=0}^{8} \mathbf{e}_{i} \mathbf{e}_{i} f_{i}^{e q}(\mathbf{x}, t)=C_{s}^{2} \rho(\mathbf{x}, t) \mathbf{I}_{d}, \\
\sum_{i=0}^{8} g_{i}^{e q}(\mathbf{x}, r ; t)=0, \\
\sum_{i=0}^{8} \mathbf{e}_{i} g_{i}^{e q}(\mathbf{x}, r ; t)=\left(\begin{array}{l}
0 \\
0
\end{array}\right), \\
\sum_{i=0}^{8} \mathbf{e}_{i} \mathbf{e}_{i} g_{i}^{e q}(\mathbf{x}, r ; t)=C_{s}^{2} \tilde{\rho}_{e}(\mathbf{x}, r ; t) \mathbf{I}_{d}, \\
\sum_{i=0}^{8} h_{i}^{e q}(\mathbf{x}, s ; t)=0 \\
\sum_{i=0}^{8} \mathbf{e}_{i} h_{i}^{e q}(\mathbf{x}, s ; t)=\left(\begin{array}{l}
0 \\
0
\end{array}\right), \\
\sum_{i=0}^{8} \mathbf{e}_{i} \mathbf{e}_{i} h_{i}^{e q}(\mathbf{x}, s ; t)=C_{s}^{2} \tilde{\rho}_{T}(\mathbf{x}, s ; t) \mathbf{I}_{d} .
\end{gathered}
$$

Remark 3.5 .

There is no uniqueness of equilibrium function definition. According to implementation choices and type of PDE, we can choose more complex forms which are more efficient in term of implementation. This choice only depends on the definitions of link functions $S_{i}^{1}$ and $S_{i}^{2}$, and time relaxations $\tau_{1}$ and $\tau_{2}$. Indeed, we can take into account nonlinear diffusion operators in relaxation terms $\tau_{1}$ and $\tau_{2}$, and we can merge equilibrium and link functions (see e.g. [34]).

Then, we can define the source terms $F_{i}, G_{i}$ and $H_{i}$, which are discrete approximations of nonlinear operator and source terms of system (14), as follows $(\forall i=0, \ldots, 8)$

$$
\begin{array}{r}
F_{i}(\mathbf{x}, t)=w_{i} F\left(\mathbf{x}, t ; \rho, \rho_{e}\right), \\
G_{i}(\mathbf{x}, r ; t)=w_{i} \tilde{G}\left(\mathbf{x}, r ; t, \rho, \tilde{\rho}_{e}\right), \\
H_{i}(\mathbf{x}, s ; t)=w_{i} \tilde{H}\left(\mathbf{x}, s ; t, \tilde{\rho}_{T}\right) .
\end{array}
$$


According again to (25) and (26), we can derive the following relations

$$
\begin{array}{r}
\sum_{i=0}^{8} F_{i}(\mathbf{x}, t)=F\left(\mathbf{x}, t ; \rho, \rho_{e}\right), \\
\sum_{i=0}^{8} \mathbf{e}_{i} F_{i}(\mathbf{x}, t)=\left(\begin{array}{l}
0 \\
0
\end{array}\right), \\
\sum_{i=0}^{8} G_{i}(\mathbf{x}, r ; t)=\tilde{G}\left(\mathbf{x}, r ; t, \rho, \tilde{\rho}_{e}\right), \\
\sum_{i=0}^{8} \mathbf{e}_{i} G_{i}(\mathbf{x}, r ; t)=\left(\begin{array}{l}
0 \\
0
\end{array}\right), \\
\sum_{i=0}^{8} H_{i}(\mathbf{x}, t)=\tilde{H}\left(\mathbf{x}, s ; t, \tilde{\rho}_{T}\right), \\
\sum_{i=0}^{8} \mathbf{e}_{i} H_{i}(\mathbf{x}, s ; t)=\left(\begin{array}{l}
0 \\
0
\end{array}\right) .
\end{array}
$$

Similarly, we define the first link function $S_{i}^{1}$ associate to the first equation of (14) and equation $(28)$ by $(\forall i=0, \ldots, 8)$

$$
S_{i}^{1}\left(\mathbf{x}, t ; \nabla \rho, \nabla \rho_{e}\right)=w_{i}\left(\beta_{\rho}(\mathbf{x}, t) \mathbf{e}_{i} \cdot \nabla \rho_{e}(\mathbf{x}, t)+\alpha_{\rho}(\mathbf{x}, t) \mathbf{e}_{i} \cdot \nabla \rho(\mathbf{x}, t)\right),
$$

and the second link function $S_{i}^{2}$ associate to the second equation of (14) and equation $(29)$ as $(\forall i=0, \ldots, 8)$

$$
S_{i}^{2}\left(\mathbf{x}, r ; t, \nabla \rho, \nabla \tilde{\rho}_{e}\right)=w_{i}\left(\beta_{\rho_{e}}(\mathbf{x}, t) \mathbf{e}_{i} \cdot \nabla \rho(\mathbf{x}, t)+\alpha_{\rho_{e}}(\mathbf{x}, t) \mathbf{e}_{i} \cdot \nabla \tilde{\rho}_{e}(\mathbf{x}, r ; t)\right),
$$

where we choose $\alpha_{\rho}, \alpha_{\rho_{e}}, \beta_{\rho}, \beta_{\rho_{e}}$ to be:

$$
\begin{gathered}
\alpha_{\rho}(\mathbf{x}, t)=-\frac{1}{\tau_{1}(\mathbf{x}, t) C_{s}^{2} \Delta t}\left(K_{i c}(\mathbf{x}, t)+C_{s}^{2} \Delta t\left(\frac{1}{2}-\tau_{1}(\mathbf{x}, t)\right)\right), \\
\beta_{\rho}(\mathbf{x}, t)=\frac{-K_{i c}(\mathbf{x}, t)}{\tau_{1}(\mathbf{x}, t) C_{s}^{2} \Delta t}, \\
\alpha_{\rho_{e}}(\mathbf{x}, t)=-\frac{1}{\tau_{2}(\mathbf{x}, t) C_{s}^{2} \Delta r}\left(\left(K_{i c}(\mathbf{x}, t)+K_{e c}(\mathbf{x}, t)\right)+C_{s}^{2} \Delta r\left(\frac{1}{2}-\tau_{2}(\mathbf{x}, t)\right)\right), \\
\beta \rho_{e}(\mathbf{x}, t)=\frac{-K_{i c}(\mathbf{x}, t)}{\tau_{2}(\mathbf{x}, t) C_{s}^{2} \Delta r} .
\end{gathered}
$$

\section{Remark 3.6 .}

As values $\alpha_{\rho}$ and $\alpha_{\rho_{e}}$ defined by the equations (61) and (64) depend on relaxation factors $\tau_{1}$ and $\tau_{2}$, we can choose them as arbitrary nonzero constants. However, for the relaxation time $\tau_{3}$, in order to recover relaxation tensor $K_{T} \mathbf{I}_{d}$ in the governing elliptic equation, we can define it as

$$
\tau_{3}(\mathbf{x}, t)=\frac{K_{T}(\mathbf{x}, t)}{C_{s}^{2} \Delta s}+\frac{1}{2} .
$$

By using the relations (25) and (26), we can deduce the following properties

$$
\begin{aligned}
\sum_{i=0}^{8} S_{i}^{1}\left(\mathbf{x}, t ; \nabla \rho, \nabla \rho_{e}\right) & =0, \\
\sum_{i=0}^{8} S_{i}^{2}\left(\mathbf{x}, r ; t, \nabla \rho, \nabla \tilde{\rho}_{e}\right) & =0,
\end{aligned}
$$




$$
\begin{aligned}
\sum_{i=0}^{8} \mathbf{e}_{i} S_{i}^{1}\left(\mathbf{x}, t, \nabla \rho, \nabla \rho_{e}\right) & =C_{s}^{2}\left(\beta_{\rho}(\mathbf{x}, t) \nabla \rho_{e}(\mathbf{x}, t)+\alpha_{\rho}(\mathbf{x}, t) \nabla \rho(\mathbf{x}, t)\right), \\
\sum_{i=0}^{8} \mathbf{e}_{i} S_{i}^{2}\left(\mathbf{x}, r ; t, \nabla \rho, \nabla \tilde{\rho}_{e}\right) & =C_{s}^{2}\left(\beta_{\rho_{e}}(\mathbf{x}, t) \nabla \rho(\mathbf{x}, t)+\alpha_{\rho_{e}}(\mathbf{x}, t) \nabla \tilde{\rho}_{e}(\mathbf{x}, r ; t)\right) .
\end{aligned}
$$

Remark 3.7 .

The link functions $S_{i}^{1}$ and $S_{i}^{2}$ depend on the gradient of functions $\rho, \rho_{e}$ and $\tilde{\rho}_{e}$. The approximation of these gradients can be directly derived from Chapman-Enskog expansion with distribution functions $f_{i}$ and $g_{i}$ (see the below equations (101) and (111)), or with a finite difference approximation type method.

We can now prove formally that the above coupled LBM recovers the governing macroscopic system (14).

\section{Proposition 3.1.}

If local equilibrium distribution functions $f_{i}^{e q}, g_{i}^{e q}$ and $h_{i}^{e q}$ satisfy relations (41)-(49), source terms $F_{i}, G_{i}, H_{i}$ respect properties given by equations (53)-(58), properties (66)(69) hold for link functions $S_{i}^{1}$ and $S_{i}^{2}$, then, for $\tau_{3}=\frac{K_{T}}{C_{s}^{2} \Delta t}+\frac{1}{2}$, the above coupled $L B M$ recover correctly macroscopic system (14) through the Chapman-Enskog analysis.

Proof: To derive the macroscopic equation from developed coupled lattice BGK model, the Chapman-Enskog expansion is applied under the assumption of small Kundsen number $\epsilon$ (which can be a ratio between a characteristic length $L$ and the particular mean free path $l$ ) to determine equilibrium functions, source terms and link functions. Each function is decomposed around its equilibrium state with different scale of perturbations. Then, the difference between associated LBE and Taylor series of the distribution function lead us to recover the governing macroscopic equation.

Before to prove the main result of proposition, we introduce the main steps of demonstration with applying Chapman-Enskog expansion to distribution functions $\phi_{i}$ of LBE (21) in order to recover governing reaction-diffusion equation (15). The macroscopic variable $\Theta$ is defined in terms of distribution functions as

$$
\begin{aligned}
& \Theta=\sum_{i=1}^{8} \phi_{i}=\sum_{i=1}^{8} \phi_{i}^{e q}, \text { with } \\
& \sum_{i=1}^{8} \mathbf{e}_{i} \phi_{i}^{e q}=\left(\begin{array}{l}
0 \\
0
\end{array}\right) \text { and } \\
& \sum_{i=0}^{8} \mathbf{e}_{i} \mathbf{e}_{i} \phi_{i}^{e q}(\mathbf{x}, t)=C_{s}^{2} \Theta(\mathbf{x}, t) \mathbf{I}_{d} .
\end{aligned}
$$

For every distribution functions $\phi_{i}$ the Chapman-Enskog expansion is applied with the small parameter $\epsilon$ as:

$$
\begin{aligned}
\phi_{i} & =\phi_{i}^{(0)}+\varepsilon \phi_{i}^{(1)}+\varepsilon^{2} \phi_{i}^{(2)}+\ldots \\
& =\phi_{i}^{e q}+\varepsilon \phi_{i}^{(1)}+\varepsilon^{2} \phi_{i}^{(2)}+\ldots \\
\frac{\partial}{\partial t} & =\varepsilon \frac{\partial}{\partial t_{1}}+\varepsilon^{2} \frac{\partial}{\partial t_{2}} \\
\nabla & =\varepsilon \nabla_{1}, \operatorname{div}_{1}=\nabla_{1}
\end{aligned}
$$

where $t_{i}$ is the time scale, for $i=1,2, \mathbf{x}_{1}$ is the space scale and $\nabla_{1}=\nabla_{\mathbf{x}_{1}}$ is the gradient with respect to $\mathbf{x}_{1}$, and we assume that every source term $\Phi_{i}$ take the form

$$
\begin{aligned}
& \Phi_{i}=\varepsilon \Phi_{i}^{(1)}+\varepsilon^{2} \Phi_{i}^{(2)}, \quad \text { with } \\
& \sum_{i=0}^{8} \Phi_{i}^{(n)}(\mathbf{x}, t)=\Phi^{(n)}(\mathbf{x}, t) \text { and } \\
& \sum_{i=0}^{8} \mathbf{e}_{i} \Phi_{i}^{(n)}(\mathbf{x}, t)=\left(\begin{array}{l}
0 \\
0
\end{array}\right) .
\end{aligned}
$$




\section{Remark 3.8.}

The Chapman-Enskog expansion is usually called multi-scale Chapman-Enskog expansion, because this ansatz lead us to decompose every function according to several perturbation scales. But we take into account the multi-scale nature of a biological or physical problem through the relaxation-time $\tau(\mathbf{x}, t)$ which is related to diffusion properties and nondimensionalization parameters.

\section{Property 3.1.}

The functions $\phi_{i}^{(0)}$ are associated to unperturbed state (i.e.: $\forall i=0, \ldots, 8: \phi_{i}^{(0)}=\phi_{i}^{e q}$ ). Moreover, since each scale of perturbation is linearly independent of each other, we can deduce that (for all $n \in I N^{*}$ )

$$
\sum_{i=0}^{8} \phi_{i}^{(n)}=0
$$

Then, we express the difference between (21) and Taylor series of distribution function $\phi_{i}$ moving with velocity $\mathbf{e}_{i}$. Indeed Taylor series also lead us to approximate the term $\phi_{i}\left(\mathbf{x}+\mathbf{e}_{i} \Delta t, t+\Delta t\right)$ as:

$$
\phi_{i}\left(\mathbf{x}+\mathbf{e}_{i} \Delta t, t+\Delta t\right)=\phi_{i}(\mathbf{x}, t)+\sum_{k=1}^{\infty} \frac{\Delta t^{k}}{k !}\left(\frac{\partial}{\partial t}+\mathbf{e}_{i} \cdot \nabla\right)^{k} \phi_{i}(\mathbf{x}, t)
$$

where

$$
\left(\frac{\partial}{\partial t}+\mathbf{e}_{i} \cdot \nabla\right)^{k}=\sum_{n=0}^{k}\left(\begin{array}{l}
k \\
n
\end{array}\right) \frac{\partial^{k-n}}{\partial t^{k-n}}\left(\mathbf{e}_{i} \cdot \nabla\right)^{n},
$$

with

$$
\left(\mathbf{e}_{i} \cdot \nabla\right)^{n}=\left(\mathbf{e}_{i} \cdot \nabla\right)\left(\mathbf{e}_{i} \cdot \nabla\right)^{n-1} .
$$

So, by using the expressions (74)-(73), Taylor series (76) and discrete LBE (21) become $(\forall i=0, \ldots, 8)$

$$
\begin{gathered}
\phi_{i}\left(\mathbf{x}+\mathbf{e}_{i} \Delta t, t+\Delta t\right)=\left(\phi_{i}^{(0)}(\mathbf{x}, t)+\varepsilon \phi_{i}^{(1)}(\mathbf{x}, t)+\ldots\right) \\
+\sum_{k=1}^{\infty} \frac{\Delta t^{k}}{k !}\left(\varepsilon \frac{\partial}{\partial t_{1}}+\varepsilon^{2} \frac{\partial}{\partial t_{2}}+\mathbf{e}_{i} \cdot\left(\varepsilon \nabla_{1}\right)\right)^{k}\left(\phi_{i}^{(0)}(\mathbf{x}, t)+\varepsilon \phi_{i}^{(1)}(\mathbf{x}, t)+\ldots\right)
\end{gathered}
$$

and

$$
\begin{aligned}
\phi_{i}\left(\mathbf{x}+\mathbf{e}_{i} \Delta t, t+\Delta t\right) & =\left(1-\frac{1}{\tau}\right)\left(\phi_{i}^{(0)}(\mathbf{x}, t)+\varepsilon \phi_{i}^{(1)}(\mathbf{x}, t)+\ldots\right) \\
+ & \frac{1}{\tau} \phi_{i}^{e q}(\mathbf{x}, t)+\Delta t\left(\varepsilon \Phi_{i}^{(1)}(\mathbf{x}, t)+\varepsilon^{2} \Phi_{i}^{(2)}(\mathbf{x}, t)\right) \\
+ & \frac{\Delta t^{2}}{2}\left(\varepsilon \frac{\partial}{\partial t_{1}}+\varepsilon^{2} \frac{\partial}{\partial t_{2}}+\varepsilon \mathbf{e}_{i} \cdot \nabla_{1}\right)\left(\varepsilon \Phi_{i}^{(1)}(\mathbf{x}, t)+\varepsilon^{2} \Phi_{i}^{(2)}(\mathbf{x}, t)\right) .
\end{aligned}
$$

By calculating the difference between relations (78) and (77), we can obtain

$$
\begin{aligned}
0= & \frac{1}{\tau}\left(\phi_{i}^{(0)}(\mathbf{x}, t)+\varepsilon \phi_{i}^{(1)}(\mathbf{x}, t)+\ldots\right) \\
& +\sum_{k=1}^{\infty} \frac{\Delta t^{k}}{k !}\left(\varepsilon \frac{\partial}{\partial t_{1}}+\varepsilon^{2} \frac{\partial}{\partial t_{2}}+\mathbf{e}_{i} \cdot\left(\varepsilon \nabla_{1}\right)\right)^{k}\left(\phi_{i}^{(0)}(\mathbf{x}, t)+\varepsilon \phi_{i}^{(1)}(\mathbf{x}, t)+\ldots\right) \\
& -\frac{1}{\tau} \phi_{i}^{e q}(\mathbf{x}, t)-\Delta t\left(\varepsilon \Phi_{i}^{(1)}(\mathbf{x}, t)+\varepsilon^{2} \Phi_{i}^{(2)}(\mathbf{x}, t)\right) \\
& -\frac{\Delta t^{2}}{2}\left(\varepsilon \frac{\partial}{\partial t_{1}}+\varepsilon^{2} \frac{\partial}{\partial t_{2}}+\varepsilon \mathbf{e}_{i} \cdot \nabla_{1}\right)\left(\varepsilon \Phi_{i}^{(1)}(\mathbf{x}, t)+\varepsilon^{2} \Phi_{i}^{(2)}(\mathbf{x}, t)\right)
\end{aligned}
$$


which can be rewritten as follows (according to different scales of $\varepsilon$ )

$$
0=\Psi_{i}^{(0)}(\mathbf{x}, t)+\varepsilon \Psi_{i}^{(1)}(\mathbf{x}, t)+\varepsilon^{2} \Psi_{i}^{(2)}(\mathbf{x}, t)+\ldots,
$$

where probability density functions $\Psi_{i}^{(n)}$ regroup all terms of (79) in order of $\varepsilon^{n}, n=0, \ldots$, respectively (which are partial differential equations for $n \neq 0$ )

$$
\begin{aligned}
\Psi_{i}^{(0)}= & \frac{1}{\tau}\left(\phi_{i}^{(0)}-\phi_{i}^{e q}\right)=0 \\
\Psi_{i}^{(1)}= & \Delta t\left(\frac{\partial}{\partial t_{1}}+\mathbf{e}_{i} \cdot \nabla_{1}\right) \phi_{i}^{(0)}+\frac{1}{\tau} \phi_{i}^{(1)}-\Delta t \Phi_{i}^{(1)}=0 \\
\Psi_{i}^{(2)}= & \left(\frac{\Delta t^{2}}{2}\left(\frac{\partial}{\partial t_{1}}+\mathbf{e}_{i} \cdot \nabla_{1}\right)^{2}+\Delta t \frac{\partial}{\partial t_{2}}\right) \phi_{i}^{(0)}+\Delta t\left(\frac{\partial}{\partial t_{1}}+\mathbf{e}_{i} \cdot \nabla_{1}\right) \phi_{i}^{(1)} \\
& +\frac{1}{\tau} \phi_{i}^{(2)}-\Delta t \Phi_{i}^{(2)}-\frac{\Delta t^{2}}{2}\left(\frac{\partial}{\partial t_{1}}+\mathbf{e}_{i} \cdot \nabla_{1}\right) \Phi_{i}^{(1)}=0
\end{aligned}
$$

As functions $\Psi_{i}$ are density probability functions, summation along each velocity $\mathbf{e}_{i}$ lead us to recover macroscopic functions, noted $\Psi^{(n)}$ at order $n$. Then, we recover macroscopic functions with

$$
\sum_{i=0}^{8}\left(\Psi_{i}^{(0)}+\varepsilon \Psi_{i}^{(1)}+\ldots\right)=\Psi^{(0)}+\varepsilon \Psi^{(1)}+\ldots
$$

and with an order $N$ accuracy as:

$$
\Psi^{(0)}+\varepsilon \Psi^{(1)}+\ldots+\varepsilon^{N-1} \Psi^{(N-1)}+\mathcal{O}\left(\varepsilon^{N}\right)=\Psi+\mathcal{O}\left(\varepsilon^{N}\right) .
$$

Finally, by retaining terms up to $\mathcal{O}\left(\varepsilon^{3}\right)$, taking (82) times $\epsilon$ plus (83) times $\epsilon^{2}$, summing over $i$ and using (81), (70), (72), (73) and (75), we can obtain, by a suitable choice of equilibrium distribution $\phi_{i}^{e q}$ (with similar properties as $f_{i}^{e q}, g_{i}^{e q}$ and $h_{i}^{e q}$ given in (41)(49)), source terms $\Phi_{i}$ (with similar properties as $F_{i}, G_{i}$ and $H_{i}$ given in (53)-(58)) and relaxation time $\tau(\mathbf{x}, t)=\frac{K(\mathbf{x}, t)}{C_{s}^{2} \Delta t}+\frac{1}{2}$, the approximation of the general reaction-diffusion equation (15).

In fact, according to (74),(70), (75) and using the relation (19), we can prove easily from (82) that

$$
\Psi^{(1)}=\Delta t\left(\frac{\partial \Theta(\mathbf{x}, t)}{\partial t_{1}}-\Phi^{(1)}(\mathbf{x}, t)\right)
$$

To treat the second order terms, we rewrite $\frac{\Delta t^{2}}{2}\left(\frac{\partial}{\partial t_{1}}+\mathbf{e}_{i} \cdot \nabla_{1}\right)^{2} \phi_{i}^{e q}$ with using first order term given by (82) as:

$$
\begin{aligned}
\frac{\Delta t^{2}}{2}\left(\frac{\partial}{\partial t_{1}}+\mathbf{e}_{i} \cdot \nabla_{1}\right)^{2} \phi_{i}^{e q} & =\frac{\Delta t}{2}\left(\frac{\partial}{\partial t_{1}}+\mathbf{e}_{i} \cdot \nabla_{1}\right)\left(-\frac{1}{\tau} \phi_{i}^{(1)}+\Delta t \Phi_{i}^{(1)}\right) \\
& =-\Delta t\left(\frac{\partial}{\partial t_{1}}+\mathbf{e}_{i} \cdot \nabla_{1}\right)\left(\frac{1}{2 \tau} \phi_{i}^{(1)}\right)+\frac{\Delta t^{2}}{2}\left(\frac{\partial}{\partial t_{1}}+\mathbf{e}_{i} \cdot \nabla_{1}\right) \Phi_{i}^{(1)}
\end{aligned}
$$

and then $\Psi_{i}^{(2)}$ becomes

$$
\begin{aligned}
\Psi_{i}^{(2)}= & \Delta t\left(\frac{\partial}{\partial t_{1}}+\mathbf{e}_{i} \cdot \nabla_{1}\right)\left(\left(1-\frac{1}{2 \tau}\right) \phi_{i}^{(1)}\right)+\frac{1}{\tau} \phi_{i}^{(2)} \\
& +\Delta t\left(\frac{\partial}{\partial t_{2}}\right) \phi_{i}^{e q}-\Delta t \Phi_{i}^{(2)} .
\end{aligned}
$$

Summing over $i$ the terms of equation (87), according again to (74),(70), (75) and using the relation (19), we can deduce that

$$
\Psi^{(2)}=\Delta t\left(\frac{\partial \Theta(\mathbf{x}, t)}{\partial t_{2}}-\Phi^{(2)}+\operatorname{div}_{1}\left(\left(1-\frac{1}{2 \tau}\right) \sum_{i=0}^{8} \mathbf{e}_{i} \phi_{i}^{(1)}\right)\right) .
$$


To express $\left(1-\frac{1}{2 \tau}\right) \sum_{i=0}^{8} \mathbf{e}_{i} \phi_{i}^{(1)}$, we use relation (82) and properties $(74),(70)$ (using (19))

$$
\left(1-\frac{1}{2 \tau}\right) \sum_{i=0}^{8} \mathbf{e}_{i} \phi_{i}^{(1)}=-\tau C_{s}^{2} \Delta t\left(1-\frac{1}{2 \tau}\right) \nabla_{1} \Theta
$$

According to the expression of $\tau$ we can deduce that $\tau C_{s}^{2} \Delta t\left(1-\frac{1}{2 \tau}\right)=K$, and then

$$
\Psi^{(2)}=\Delta t\left(\frac{\partial \Theta(\mathbf{x}, t)}{\partial t_{2}}-\operatorname{div}_{1}\left(K \nabla_{1} \Theta(\mathbf{x}, t)\right)-\Phi^{(2)}\right) .
$$

Then, we can recompose $\Psi$ with using (81), (85), (86) and (88) and deduce the following approximation of equation (15) as

$$
\frac{\partial \Theta(\mathbf{x}, t)}{\partial t}-\operatorname{div}(K(\mathbf{x}, t) \nabla \Theta(\mathbf{x}, t))-\Phi(\mathbf{x}, t ; \Theta)=0
$$

Now, we will follow the previous process and steps for each equation of bidomain system (14) and its associate discrete LBE system (28)-(30). For this, we perform the Taylor expansion on (28)-(30) up to terms with order of $\mathcal{O}\left(\varepsilon^{3}\right)$ and we use ansatz given by equations (71)-(73) where distribution functions $f_{i}, g_{i}$ and $h_{i}$ successively replace $\phi_{i}$ with order 2 expansion given by equation (71) and we assume that the source terms and link functions $F_{i}, G_{i}, H_{i}, S_{i}^{1}$ and $S_{i}^{2}$ take the form (which are defined like source term $\Phi_{i}$ with order 2 expansion)

$$
\begin{aligned}
F_{i} & =\varepsilon F_{i}^{(1)}+\varepsilon^{2} F_{i}^{(2)}, \text { with } \sum_{i=0}^{8} \mathbf{e}_{i} F_{i}^{(j)}=0, \text { for } j=1,2, \\
S_{i}^{1} & =\varepsilon S_{i}^{1,(1)}, \text { with } \sum_{i=0}^{8} S_{i}^{1,(1)}=0, \\
G_{i} & =\varepsilon G_{i}^{(1)}+\varepsilon^{2} G_{i}^{(2)}, \text { with } \sum_{i=0}^{8} \mathbf{e}_{i} G_{i}^{(j)}=0, \text { for } j=1,2, \\
S_{i}^{2} & =\varepsilon S_{i}^{2,(1)}, \text { with } \sum_{i=0}^{8} S_{i}^{2,(1)}=0, \\
H_{i} & =\varepsilon H_{i}^{(1)}+\varepsilon^{2} H_{i}^{(2)}, \text { with } \sum_{i=0}^{8} \mathbf{e}_{i} H_{i}^{(j)}=0, \text { for } j=1,2 .
\end{aligned}
$$

First, we consider the first equation of (14) and its associate discrete LBE system (28). According to (90)-(91) and using similar arguments to derive the terms $\Psi_{i}^{(n)}$ in (81)-(87), we can obtain the following terms $\mathcal{P}_{i}^{(n)}, n=0,2$ (after division by $\Delta t$ )

$$
\begin{aligned}
\mathcal{P}_{i}^{(0)}= & -\frac{1}{\Delta t \tau_{1}}\left(f_{i}^{(0)}-f_{i}^{e q}\right)=0 \\
\mathcal{P}_{i}^{(1)}= & \left(\frac{\partial}{\partial t_{1}}+\mathbf{e}_{i} \cdot \nabla_{1}\right) f_{i}^{e q}+\frac{1}{\tau_{1} \Delta t} f_{i}^{(1)} \\
& -S_{i}^{1,(1)}-F_{i}^{(1)}=0 \\
\mathcal{P}_{i}^{(2)}= & \left(\frac{\partial}{\partial t_{1}}+\mathbf{e}_{i} \cdot \nabla_{1}\right) f_{i}^{(1)} \\
& +\left(\frac{\partial}{\partial t_{2}}+\frac{\Delta t}{2}\left(\frac{\partial}{\partial t_{1}}+\mathbf{e}_{i} \cdot \nabla_{1}\right)^{2}\right) f_{i}^{e q} \\
& +\frac{1}{\tau_{1} \Delta t} f_{i}^{(2)}-\frac{\Delta t}{2}\left(\frac{\partial}{\partial t_{1}}+\mathbf{e}_{i} \cdot \nabla_{1}\right) F_{i}^{(1)}-F_{i}^{(2)}=0 .
\end{aligned}
$$


Summing over $i$ the terms of equation (96) and using the fact that $\sum_{i=0}^{8} f_{i}^{(n)}=0$, for $n \neq 0$, and the relations (41), (42), (53), (66), (90) and (91) we obtain

$$
\epsilon \mathcal{P}^{(1)}=\epsilon \sum_{i=0}^{8} \mathcal{P}_{i}^{(1)}=\epsilon \frac{\partial \rho}{\partial t_{1}}-\varepsilon F^{(1)}=0 .
$$

By rewriting the equation (97) with the equation (96), we obtain

$$
\begin{aligned}
\mathcal{P}_{i}^{(2)}= & \left(\frac{\partial}{\partial t_{1}}+\mathbf{e}_{i} \cdot \nabla_{1}\right)\left(\left(1-\frac{1}{2 \tau_{1}}\right) f_{i}^{(1)}\right) \\
& +\frac{\partial f_{i}^{e q}}{\partial t_{2}}+\frac{1}{\tau_{1} \Delta t} f_{i}^{(2)} \\
& +\frac{\Delta t}{2}\left(\frac{\partial}{\partial t_{1}}+\mathbf{e}_{i} \cdot \nabla_{1}\right) S_{i}^{1,(1)}-F_{i}^{(2)} .
\end{aligned}
$$

Summing over $i$ the terms of equation (99) and using the fact that $\sum_{i=0}^{8} f_{i}^{(n)}=0$, for $n \neq 0$, Remark 3.2 and the relations (41), (42), (53), (66), (90) and (91) we obtain

$$
\begin{aligned}
\epsilon^{2} \mathcal{P}^{(2)}=\epsilon^{2} \sum_{i=0}^{8} \mathcal{P}_{i}^{(2)}= & \epsilon^{2} \frac{\partial \rho}{\partial t_{2}}+\operatorname{div}\left(\epsilon\left(1-\frac{1}{2 \tau_{1}}\right) \sum_{i=0}^{8} \mathbf{e}_{i} f_{i}^{(1)}\right) \\
& +\frac{\Delta t}{2}\left(\operatorname{div}\left(\sum_{i=0}^{8} \mathbf{e}_{i} S_{i}^{1}\right)\right)-\epsilon^{2} F^{(2)},
\end{aligned}
$$

with $F^{(j)}=\sum_{i=0}^{8} F_{i}^{(j)}$, for $j=1,2$.

Now we calculate $\left(1-\frac{1}{2 \tau_{1}}\right) \epsilon \sum_{i=0}^{8} \mathbf{e}_{i} f_{i}^{(1)}$. From (96) (to express $\sum_{i=0}^{8} \mathbf{e}_{i} f_{i}^{(1)}$ ) and according to (19), (54), (68), (90) and (91), we can deduce

$$
\left(1-\frac{1}{2 \tau_{1}}\right) \epsilon \sum_{i=0}^{8} \mathbf{e}_{i} f_{i}^{(1)}=-\left(1-\frac{1}{2 \tau_{1}}\right) C_{s}^{2} \tau_{1} \Delta t \nabla \rho+\tau_{1} \Delta t\left(1-\frac{1}{2 \tau_{1}}\right) \sum_{i=0}^{8} \mathbf{e}_{i} S_{i}^{1} .
$$

According to (68) and (101), the relation (100) becomes

$$
\begin{aligned}
\epsilon^{2} \mathcal{P}^{(2)}= & \epsilon^{2} \frac{\partial \rho}{\partial t_{2}}-\epsilon^{2} F^{(2)} \\
& +\operatorname{div}\left(C_{s}^{2} \tau_{1} \Delta t\left(\beta_{\rho} \nabla \rho_{e}+\left(\alpha_{\rho}+\left(1-\frac{1}{2 \tau_{1}}\right)\right) \nabla \rho\right)\right) .
\end{aligned}
$$

Then (from (59), (61) and (62))

$$
\epsilon^{2} \mathcal{P}^{(2)}=\epsilon^{2} \frac{\partial \rho}{\partial t_{2}}-\epsilon^{2} F^{(2)}-\operatorname{div}\left(K_{i c}\left(\nabla \rho+\nabla \rho_{e}\right)\right) .
$$

Consequently, from (98) and (103), we can recover the macroscopic parabolic equation of (14) with

$$
0=\mathcal{P}^{(0)}+\varepsilon \mathcal{P}^{(1)}+\varepsilon^{2} \mathcal{P}^{(2)}+\mathcal{O}\left(\varepsilon^{3}\right)=\frac{\partial \rho}{\partial t}-F-\operatorname{div}\left(K_{i c}\left(\nabla \rho+\nabla \rho_{e}\right)\right) .
$$

Second, for a fixed time $t$, we consider the second equation of (14) and its associate discrete pseudo-time-stepping LBM system (29) (for pseudo-time variables $r$ with $\frac{\partial}{\partial r}=$ 
$\varepsilon \frac{\partial}{\partial r_{1}}+\varepsilon^{2} \frac{\partial}{\partial r_{2}}$ and pseudo-time step $\left.\Delta r\right)$. By using similar arguments to derive the terms $\mathcal{P}_{i}^{(n)}, n=0,2$, we can obtain the following terms (after division by $\Delta r$ )

$$
\begin{aligned}
\mathcal{Q}_{i}^{(0)}= & -\frac{1}{\Delta t \tau_{2}}\left(g_{i}^{(0)}-g_{i}^{e q}\right)=0 \\
\mathcal{Q}_{i}^{(1)}= & \left(\frac{\partial}{\partial r_{1}}+\mathbf{e}_{i} \cdot \nabla_{1}\right) g_{i}^{e q} \\
& +\frac{1}{\tau_{2} \Delta r} g_{i}^{(1)}-S_{i}^{2,(1)}-G_{i}^{(1)}=0 \\
\mathcal{Q}_{i}^{(2)=} & \left(\frac{\partial}{\partial r_{1}}+\mathbf{e}_{i} \cdot \nabla_{1}\right) g_{i}^{(1)} \\
& +\left(\frac{\partial}{\partial r_{2}}+\frac{\Delta r}{2}\left(\frac{\partial}{\partial r_{1}}+\mathbf{e}_{i} \cdot \nabla_{1}\right)^{2}\right) g_{i}^{e q} \\
& +\frac{1}{\tau_{2} \Delta r} g_{i}^{(2)}-\frac{\Delta r}{2}\left(\frac{\partial}{\partial r_{1}}+\mathbf{e}_{i} \cdot \nabla_{1}\right) G_{i}^{(1)}-G_{i}^{(2)}=0 .
\end{aligned}
$$

Summing over $i$ the terms of equation (106) and using the fact that $\sum_{i=0}^{8} g_{i}^{(n)}=0$, for $n \neq 0$, and the relations (44), (45) and (93) we obtain

$$
\mathcal{Q}^{(1)}=-\tilde{G}^{(1)},
$$

with $\tilde{G}^{(1)}=\sum_{i=0}^{8} G_{i}^{(1)}$.

By rewriting the equation (107) with the equation (106), we obtain

$$
\begin{aligned}
\mathcal{Q}_{i}^{(2)}= & \left(\frac{\partial}{\partial r_{1}}+\mathbf{e}_{i} \cdot \nabla_{1}\right)\left(\left(1-\frac{1}{2 \tau_{2}}\right) g_{i}^{(1)}\right) \\
& +\frac{\partial g_{i}^{e q}}{\partial r_{2}}+\frac{1}{\tau_{2} \Delta r} g_{i}^{(2)} \\
& +\frac{\Delta r}{2}\left(\frac{\partial}{\partial r_{1}}+\mathbf{e}_{i} \cdot \nabla\right) S_{i}^{2,(1)}-G_{i}^{(2)} .
\end{aligned}
$$

Summing over $i$ the terms of equation (109) and using the fact that $\sum_{i=0}^{8} g_{i}^{(n)}=0$, for $n \neq 0, \sum_{i=0}^{8} g_{i}^{e q}=0$, Remark 3.2 and the relations (44), (45)and (93) we obtain

$$
\begin{aligned}
\epsilon^{2} \mathcal{Q}^{(2)}=\epsilon^{2} \sum_{i=0}^{8} \mathcal{Q}_{i}^{(2)}= & \operatorname{div}\left(\epsilon\left(1-\frac{1}{2 \tau_{2}}\right) \sum_{i=0}^{8} \mathbf{e}_{i} g_{i}^{(1)}\right) \\
& +\frac{\Delta r}{2}\left(\operatorname{div}\left(\sum_{i=0}^{8} \mathbf{e}_{i} S_{i}^{2}\right)\right)-\epsilon^{2} \tilde{G}^{(2)}
\end{aligned}
$$

with $\tilde{G}^{(2)}=\sum_{i=0}^{8} G_{i}^{(2)}$.

Now we calculate $\left(1-\frac{1}{2 \tau_{2}}\right) \epsilon \sum_{i=0}^{8} \mathbf{e}_{i} g_{i}^{(1)}$. From (106) and according to (19), (54), (69), (92) and (93), we can deduce

$$
\left(1-\frac{1}{2 \tau_{2}}\right) \epsilon \sum_{i=0}^{8} \mathbf{e}_{i} g_{i}^{(1)}=-\left(1-\frac{1}{2 \tau_{2}}\right) C_{s}^{2} \tau_{2} \Delta r \nabla \tilde{\rho}+\tau_{2} \Delta r\left(1-\frac{1}{2 \tau_{1}}\right) \sum_{i=0}^{8} \mathbf{e}_{i} S_{i}^{2} .
$$


According to (69) and (111), the relation (110) becomes

$$
\begin{aligned}
\epsilon^{2} \mathcal{Q}^{(2)}= & -\epsilon^{2} \tilde{G}^{(2)} \\
& +\operatorname{div}\left(C_{s}^{2} \tau_{2} \Delta r\left(\beta_{\rho_{e}} \nabla \rho+\left(\alpha_{\rho_{e}}+\left(1-\frac{1}{2 \tau_{2}}\right)\right) \nabla \tilde{\rho}_{e}\right)\right) .
\end{aligned}
$$

Then (from (60), (63) and (64))

$$
\epsilon^{2} \mathcal{Q}^{(2)}=-\epsilon^{2} \tilde{G}^{(2)}-\operatorname{div}\left(K_{i c} \nabla \rho+\left(K_{i c}+K_{e c}\right) \nabla \tilde{\rho}_{e}\right) .
$$

We can now sum the terms of order 0 to 2 to obtain the following equation (from (108) and (113))

$$
0=\mathcal{Q}^{(0)}+\varepsilon \mathcal{Q}^{(1)}+\varepsilon^{2} \mathcal{Q}^{(2)}+\mathcal{O}\left(\varepsilon^{3}\right)=-\tilde{G}-\operatorname{div}\left(K_{i c} \nabla \rho+\left(K_{i c}+K_{e c}\right) \nabla \tilde{\rho}_{e}\right) .
$$

To recover the first elliptic equation of system (14), we use the two limits (34) and (36) to write the following limit: (formally)

$$
\begin{aligned}
\lim _{r \rightarrow \infty} & \left(-\tilde{G}\left(\mathbf{x}, r ; t, \rho, \rho_{e}\right)-\operatorname{div}\left(K_{i c} \nabla \rho(\mathbf{x}, t)+\left(K_{i c}+K_{e c}\right) \nabla \tilde{\rho}_{e}(\mathbf{x}, r ; t)\right)\right) \\
= & -G\left(\mathbf{x}, t ; \rho, \rho_{e}\right)-\operatorname{div}\left(K_{i c} \nabla \rho(\mathbf{x}, t)+\left(K_{i c}+K_{e c}\right) \nabla \rho_{e}(\mathbf{x}, t)\right) .
\end{aligned}
$$

Finally, we apply the previous method to recover the governing second elliptic equation of the system (14). For that, for a fixed time $t$, we consider the discrete pseudo-timestepping LBM system (30) (for pseudo-time variables $s$ with $\frac{\partial}{\partial s}=\varepsilon \frac{\partial}{\partial s_{1}}+\varepsilon^{2} \frac{\partial}{\partial s_{2}}$ and pseudo-time step $\Delta s$ ). By using similar arguments to derive the terms $\mathcal{Q}_{i}^{(n)}, n=0,2$, we can obtain the following terms (after division by $\Delta s$ )

$$
\begin{aligned}
\mathcal{R}_{i}^{(0)}= & -\frac{1}{\tau_{3} \Delta s}\left(h_{i}^{(0)}-h_{i}^{e q}\right)=0 \\
\mathcal{R}_{i}^{(1)}= & \left(\frac{\partial}{\partial s_{1}}+\mathbf{e}_{i} \cdot \nabla_{1}\right) h_{i}^{e q}+\frac{1}{\tau_{3} \Delta s} h_{i}^{(1)}-H_{i}^{(1)}=0 \\
\mathcal{R}_{i}^{(2)}= & \left(\frac{\partial}{\partial s_{1}}+\mathbf{e}_{i} \cdot \nabla_{1}\right) h_{i}^{(1)} \\
& +\left(\frac{\partial}{\partial s_{2}}+\frac{\Delta s}{2}\left(\frac{\partial}{\partial s_{1}}+\mathbf{e}_{i} \cdot \nabla_{1}\right)^{2}\right) h_{i}^{e q} \\
& +\frac{1}{\tau_{3} \Delta s} h_{i}^{(2)}-\frac{\Delta s}{2}\left(\frac{\partial}{\partial s_{1}}+\mathbf{e}_{i} \cdot \nabla_{1}\right) H_{i}^{(1)}-H_{i}^{(2)}=0 .
\end{aligned}
$$

Summing over $i$ the terms of equation (117) and using the fact that $\sum_{i=0}^{8} h_{i}^{(n)}=0$, for $n \neq 0$, and the relations $(47)$ and (48) we obtain

$$
\varepsilon \mathcal{R}^{(1)}=-\varepsilon \tilde{H}^{(1)}
$$

with $\tilde{H}^{(1)}=\sum_{i=0}^{8} H_{i}^{(1)}$.

By rewriting the equation (118) with the equation (117), we obtain

$$
\begin{aligned}
\mathcal{R}_{i}^{(2)}= & \left(\frac{\partial}{\partial s_{1}}+\mathbf{e}_{i} \cdot \nabla_{1}\right)\left(\left(1-\frac{1}{2 \tau_{3}}\right) h_{i}^{(1)}\right) \\
& +\frac{\partial h_{i}^{e q}}{\partial s_{2}}+\frac{1}{\tau_{3} \Delta r} h_{i}^{(2)} \\
& -H_{i}^{(2)} .
\end{aligned}
$$


Summing over $i$ the terms of equation (120) and using the fact that $\sum_{i=0}^{8} h_{i}^{(n)}=0$, for $n \neq 0, \sum_{i=0}^{8} h_{i}^{e q}=0$, Remark 3.2 and the relations (47) and (48) we obtain

$$
\begin{gathered}
\epsilon^{2} \mathcal{R}^{(2)}=\epsilon^{2} \sum_{i=0}^{8} \mathcal{R}_{i}^{(2)}=\operatorname{div}_{-\epsilon^{2} \tilde{H}^{(2)}}^{\left(\epsilon\left(1-\frac{1}{2 \tau_{3}}\right)\right.} \\
\left.\sum_{i=0}^{8} \mathbf{e}_{i} h_{i}^{(1)}\right)
\end{gathered}
$$

with $\tilde{H}^{(2)}=\sum_{i=0}^{8} H_{i}^{(2)}$.

Now we calculate $\left(1-\frac{1}{2 \tau_{3}}\right) \epsilon \sum_{i=0}^{8} \mathbf{e}_{i} h_{i}^{(1)}$. From (117) and according to (19), (58) and (65), we can deduce

$$
\left(1-\frac{1}{2 \tau_{3}}\right) \epsilon \sum_{i=0}^{8} \mathbf{e}_{i} h_{i}^{(1)}=-\left(1-\frac{1}{2 \tau_{3}}\right) C_{s}^{2} \tau_{3} \Delta s \nabla \tilde{\rho}_{T}=-K_{T} \nabla \tilde{\rho}_{T} .
$$

According to (122), the relations (121) becomes

$$
\epsilon^{2} \mathcal{R}^{(2)}=-\epsilon^{2} \tilde{H}^{(2)}-\operatorname{div}\left(K_{T} \nabla \tilde{\rho}_{T}\right) .
$$

We can now sum the terms of order 0 to 2 to obtain the following equation (from (119) and (123))

$$
0=\mathcal{R}^{(0)}+\varepsilon \mathcal{R}^{(1)}+\varepsilon^{2} \mathcal{R}^{(2)}+\mathcal{O}\left(\varepsilon^{3}\right)=-\tilde{H}-\operatorname{div}\left(K_{T} \nabla \tilde{\rho}_{T}\right)
$$

To recover the second elliptic equation of system (14), we use the two limits (35) and (37) to write the following limit (formally):

$$
\begin{aligned}
\lim _{s \rightarrow \infty} & \left(-\tilde{H}\left(\mathbf{x}, s ; t, \rho_{T}\right)-\operatorname{div}\left(K_{T} \nabla \tilde{\rho}_{T}(\mathbf{x}, s ; t)\right)\right) \\
& =-H\left(\mathbf{x}, t ; \rho_{T}\right)-\operatorname{div}\left(K_{T} \nabla \rho_{T}(\mathbf{x}, t)\right) .
\end{aligned}
$$

This completes the proof.

\subsection{Treatment of ODE}

Now, we present briefly the method to solve ODE satisfy by variable $V$ on the interval $\left[s_{1}, s_{2}\right]$, with initial condition $V\left(\mathbf{x}, s_{1}\right)=V_{0}(\mathbf{x})$. As:

$$
\frac{\partial V(\mathbf{x}, s)}{\partial s}=W(\mathbf{x}, s ; V)
$$

where operator $W$ is defined by

$$
W(\mathbf{x}, s ; V)=\tilde{W}(\mathbf{x}, s)-\lambda(\mathbf{x}) V(\mathbf{x}, s) .
$$

By using Gronwall Lemma we can deduce that

$$
V\left(\mathbf{x}, s_{2}\right)=V_{0}(\mathbf{x}) e^{-\lambda(\mathbf{x})\left(s_{2}-s_{1}\right)}+e^{-\lambda(\mathbf{x}) s_{1}} \int_{s_{1}}^{s_{2}} \tilde{W}(\mathbf{x}, s) e^{\lambda(\mathbf{x}) s} d s .
$$

Then, according to approximation of derived integral by trapezoidal method between $t_{1}$ and $t_{2}$ we obtain the following approximation of $V$ denoted also by $V$

$$
V\left(., s_{2}\right)=V_{0}(.) e^{-\lambda(.)\left(s_{2}-s_{1}\right)}+\frac{s_{2}-s_{1}}{2}\left(\tilde{W}\left(., s_{1}\right)+\tilde{W}\left(., s_{2}\right) e^{\lambda(.)\left(s_{2}-s_{1}\right)}\right) .
$$

In our case, we assume that for all $t \in[0 ; T-\Delta t]$ the ionic function $U$ given by (3) looks like $V$ given by (127) between $t$ and $t+\Delta t$. So with thin enough discretization, we aim to reach an order 2 convergence space accuracy method to approximate ionic variable $U$ with using relation (128). 


\subsection{Algorithm}

After non-dimensionalization, mesh definition and initialization of initial conditions, parameters and data, the proposed algorithm to solve the full coupled heart and torso bidomain system can be summarized as follows

1. Initialization: $\mathrm{t}=0$.

2. LBE according to time $\mathrm{t}$ by using (28) to compute $\rho(\mathbf{x}, t+\Delta t)$.

3. Trapezoidal method by using (128) to compute $U(\mathbf{x}, t+\Delta t)$.

4. Loop on new time variable $r$ :

(a) LBE according to time $\mathrm{r}$ by using (29) to compute $\tilde{\rho}_{e}(\mathbf{x}, r+\Delta r ; t+\Delta t)$ (i.e., approximation of $\left.\rho_{e}(\mathbf{x}, t+\Delta t)\right)$.

(b) If we don't reach the stopping criteria chosen to be:

$$
\left|g_{i}(\mathbf{x}, r+\Delta r ; t)-g_{i}(\mathbf{x}, r ; t)\right|<C_{\rho_{e}} \Delta \mathbf{x}^{N_{e}},
$$

where $C_{\rho_{e}}$ is a chosen constant and $N_{e}$ the order of accuracy we want, set $r=r+\Delta r$ and go back to (4a).

5. Set $\rho_{e}(\mathbf{x}, t+\Delta t):=\tilde{\rho}_{e}(\mathbf{x}, r ; t+\Delta t)$.

6. Loop on new time variable s:

(a) LBE according to time s by using (30) to compute $\tilde{\rho}_{T}(\mathbf{x}, s+\Delta s ; t+\Delta t)$ (i.e., approximation of $\left.\rho_{T}(\mathbf{x}, t+\Delta t)\right)$, with taking into account boundary condition (12) between $\rho_{T}$ and $\rho_{e}$.

(b) If we don't reach the stopping criteria chosen to be:

$$
\left|h_{i}(\mathbf{x}, s+\Delta s ; t)-h_{i}(\mathbf{x}, s ; t)\right|<C_{\rho_{T}} \Delta \mathbf{x}^{N_{T}},
$$

where $C_{\rho_{T}}$ is a chosen constant and $N_{T}$ the order of accuracy we want, set $s=s+\Delta s$ and go back to (6a).

7. Set $\rho_{T}(\mathbf{x}, t+\Delta t):=\tilde{\rho}_{T}(\mathbf{x}, s ; t+\Delta t)$.

8. If $t \neq T$, set $t:=t+\Delta t$ and go back to (2).

Remark 3.9 .

In order to parallelize the LBM, the main idea is to treat separately the collision and the streaming phases. The collision step is strictly local and can be computed on each node independently of its neighbors. Then, the streaming is just an exchange of data between its node of origin and the adjacent neighbor along velocity (see e.g. [30], [38], and references therein).

\section{Numerical simulation and applications}

To validate the capacity of our modified coupled LBM to deal with 2D bidomain systems, several situations are numerically simulated. In this study, we consider the phenomenological two-variable model proposed by Mitchell and Schaeffer [26]. The functions $H_{i o n}$ and $\mathcal{I}_{i o n}$ are given by:

$$
\begin{aligned}
\mathcal{I}_{\text {ion }} & =-\frac{U}{\mathcal{T}_{\text {in }}} \frac{\left(\rho-\rho_{\min }\right)^{2}\left(\rho_{\max }-\rho\right)}{\rho_{\max }-\rho_{\min }} \\
& +\frac{1}{\mathcal{T}_{\text {out }}} \frac{\rho-\rho_{\min }}{\rho_{\max }-\rho_{\min }}
\end{aligned}
$$


and

$$
H_{\text {ion }}= \begin{cases}\frac{U}{\mathcal{T}_{\text {open }}}-\frac{1}{\mathcal{T}_{\text {open }}\left(\rho_{\text {max }}-\rho_{\text {min }}\right)^{2}} & \text { if } \rho<\rho_{\text {gate }} \\ \frac{\mathcal{T}_{\text {close }}}{\text { los }} \rho \geq \rho_{\text {gate }}\end{cases}
$$

Then, the ionic variable $U$ depends on the change-over voltage $\rho_{\text {gate }}$, the resting potential $\rho_{\text {min }}$, the maximum potential $\rho_{\max }$, and on the time constants for opening, $\mathcal{T}_{\text {open }}$, and closing, $\mathcal{T}_{\text {close }}$. The time constants $\mathcal{T}_{\text {in }}$ and $\mathcal{T}_{\text {out }}$ are respectively related to the length of depolarization and repolarization phases. These constants are such that

$$
\mathcal{T}_{\text {in }}<\mathcal{T}_{\text {out }}<\min \left(\mathcal{T}_{\text {open }}, \mathcal{T}_{\text {close }}\right)
$$

In the sequel, we take the values of the parameters, which are consistent with the analysis and observation reported in Mitchell and Schaeffer [26], given e.g. in [7] (see Table 1). Moreover, we assume that the domain heart-torso is in a square region $\Omega=$

\begin{tabular}{ccc}
\hline Description & name & value (unit) \\
\hline Cell surface to volume ratio & $\kappa$ & $200\left(\mathrm{~cm}^{-1}\right)$ \\
transmembrane capacitance & $C_{m}$ & $10^{-3}\left(\mathrm{~F} / \mathrm{cm}^{2}\right)$ \\
Depolarization length & $\mathcal{T}_{\text {in }}$ & $4.5(\mathrm{~ms})$ \\
Repolarization length & $\mathcal{T}_{\text {out }}$ & $90(\mathrm{~ms})$ \\
Opening time constant & $\mathcal{T}_{\text {open }}$ & $100(\mathrm{~ms})$ \\
Closing time constant & $\mathcal{T}_{\text {close }}$ & $130(\mathrm{~ms})$ \\
Change-over voltage & $\rho_{\text {gate }}$ & $-67(\mathrm{mV})$ \\
Resting potential & $\rho_{\text {min }}$ & $-80(\mathrm{mV})$ \\
Maximum potential & $\rho_{\text {max }}$ & $20(\mathrm{mV})$ \\
Activation time & $T_{\text {act }}$ & $10(\mathrm{~ms})$ \\
Time-delay & $\mathcal{T}_{d}$ & $20(\mathrm{~ms})$ \\
& &
\end{tabular}

Table 1: Cell membrane parameters.

$\left[-L_{T} / 2 ; L_{T} / 2\right] \times\left[-L_{T} / 2 ; L_{T} / 2\right]=\Omega_{H} \cup \Omega_{T}$, where $\Omega_{H}=\left[0 ; L_{H}\right] \times\left[0 ; L_{H}\right]$ and $\Omega_{T}=$ $\Omega \backslash \Omega_{H}$, where $L_{H}$ and $L_{T}$ are respectively heart and torso lengths (Figure 5 ).

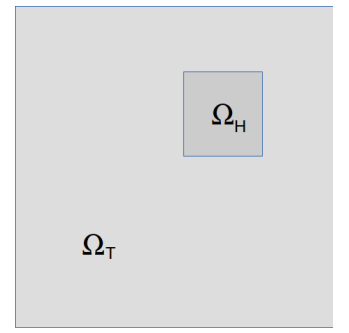

Figure 5: Heart-torso domain.

Nota Bene: If the exact solution $\phi_{\text {sol }}$ is known, we can measure the efficiency of method with the following $L^{2}$ relative error:

$$
\operatorname{Err}_{\phi}=\frac{\left\|\phi_{\text {sol }}-\phi\right\|_{L^{2}(\Omega)}}{\left\|\phi_{s o l}\right\|_{L^{2}(\Omega)}}
$$

\subsection{Benchmark problem and validation}

In this first analysis, we investigate the accuracy and spatial convergence rate of the proposed modified LBM for which we postulate that the error estimates of the method is of order 2 in space and of order 1 in time (for sufficiently regular solution). We assume 
$L_{H}=1, L_{T}=3$ and $T=1$, and we take $K_{i c}=1, K_{e c}=1, \xi_{i}=0, \xi_{e}=0, \rho(\mathbf{x}, t=0)=0$ and $U(\mathbf{x}, t=0)=\cos (\pi(x+y))$. Moreover the source terms $F_{\rho}, F_{\rho_{e}}$ and $F_{\rho_{T}}$ are added to parabolic-elliptic system (4) and elliptic equation in thorax (6), respectively, to ensure that the function $\left(\rho, \rho_{e}, U, \rho_{T}\right)$ given by (with $\left.\mathbf{x}=(x, y)\right)$ :

$$
\begin{gathered}
\rho(\mathbf{x}, t)=t x^{2}(x-1)^{2} y^{2}(y-1)^{2}, \quad \rho_{e}(\mathbf{x}, t)=t(\cos (\pi x)+\cos (\pi y)), \quad U(\mathbf{x}, t)=e^{t} \cos (\pi(x+y)) \\
\rho_{T}(\mathbf{x}, t)=K_{i c} \rho(x, y, t)+\left(K_{i c}+K_{e c}\right) \rho_{e}(x, y, t)
\end{gathered}
$$

is the exact solution of system (4)-(6) (with appropriate source term).

To study the convergence, we have constructed a sequence of meshes with decreasing spatial step $\Delta \mathrm{x}$ between $1 / 25$ and $1 / 200$ and $\Delta t=\Delta x^{2}$. Figures $6,8,7,9$ show respectively numerical solution of $\rho, \rho_{e}, U$ and $\rho_{T}$ at $t=1$ for the step $\Delta \mathbf{x}=1 / 100$.

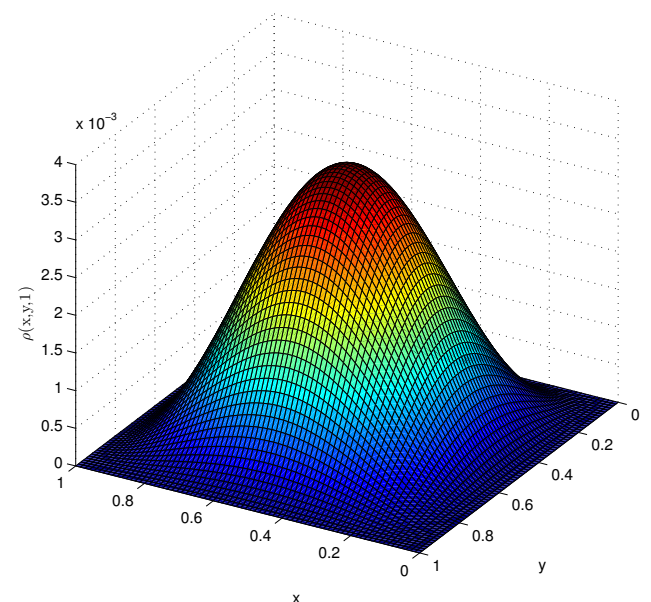

Figure 6: Potential $\rho$ in $\Omega_{H}$ at $t=1$.

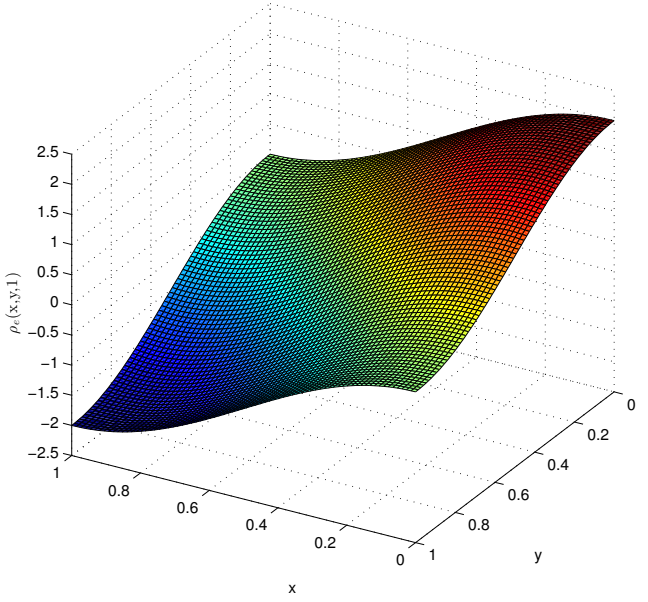

Figure 8: Potential $\rho_{e}$ in $\Omega_{H}$ at $t=1$.

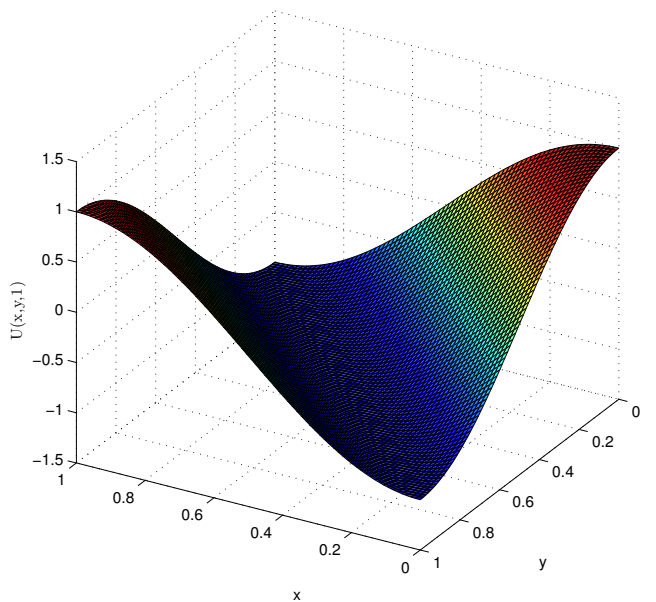

Figure 7: Ionic variable $U$ in $\Omega_{H}$ at $t=1$

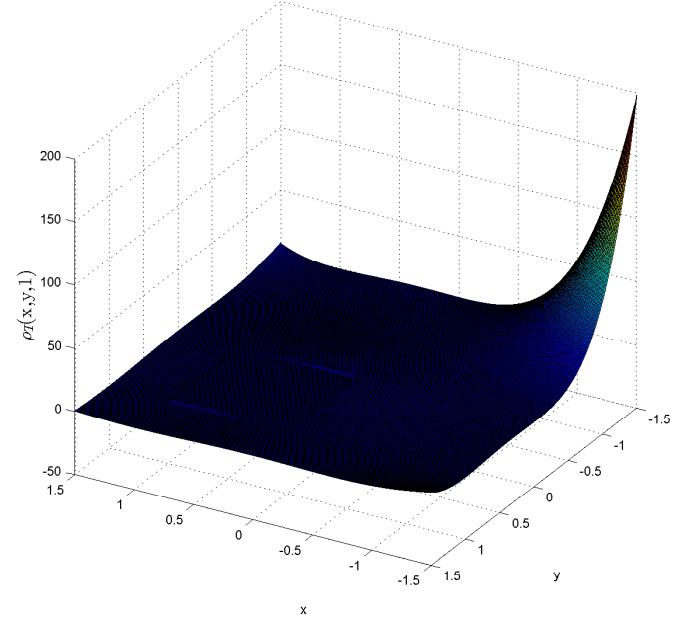

Figure 9: Potential $\rho_{T}$ in $\Omega_{T}$ at $t=1$

Since, the chosen stopping criteria for the iterative method to approach $\rho_{e}$ and $\rho_{T}$ involves a constant error (because this criteria is not defined in function of lattice size cell), then we analyze only the numerical estimate of relative error of approximation of $\rho$ and $U$. 
We present on Figures 10 and 11 (at $t=0.5$ and $t=1$ ) the convergence curves, $\log ($ Error) versus $\Delta x$, for $\rho$ and $U$ (numerical values can be found in Table 2). We observe that the slope of error curves for $\rho$ passes approximately from 1.5 to 2 and the slope of error curves for $U$ is approximately equal to 2 . This shows that our numerical error estimates agree with the postulated error estimates, and indicate the good performance of our method.

\begin{tabular}{crrc}
\hline $\mathrm{t}=0.5$ & $\Delta x$ & $\operatorname{Err}_{\rho}$ & $\operatorname{Err}_{U}$ \\
& $1 / 25$ & 0.0937 & $7.972 \times 10^{-4}$ \\
\cline { 2 - 4 } & $1 / 50$ & 0.0334 & $2.039 \times 10^{-4}$ \\
\cline { 2 - 4 } & $1 / 75$ & 0.0141 & $7.971 \times 10^{-5}$ \\
\hline $1 / 100$ & 0.0074 & $4.483 \times 10^{-5}$ \\
\hline $1 / 150$ & 0.0032 & $1.990 \times 10^{-5}$ \\
\hline $1 / 200$ & 0.0017 & $1.121 \times 10^{-5}$ \\
\hline
\end{tabular}

\begin{tabular}{cccc}
\hline $\mathrm{t}=1$ & $\Delta x$ & $\operatorname{Err}_{\rho}$ & $\operatorname{Err}_{U}$ \\
& $1 / 25$ & 0.1204 & 0.0012 \\
\cline { 2 - 4 } & $1 / 50$ & 0.0541 & $3.117 \times 10^{-4}$ \\
\cline { 2 - 4 } & $1 / 75$ & 0.0277 & $1.471 \times 10^{-4}$ \\
\hline $1 / 100$ & 0.0187 & $7.314 \times 10^{-5}$ \\
\hline $1 / 150$ & 0.0097 & $3.417 \times 10^{-5}$ \\
\hline $1 / 200$ & 0.0053 & $2.237 \times 10^{-5}$ \\
\hline
\end{tabular}

Table 2: Relation between relative error and lattice spacing for $\rho$ and $\mathrm{U}$.

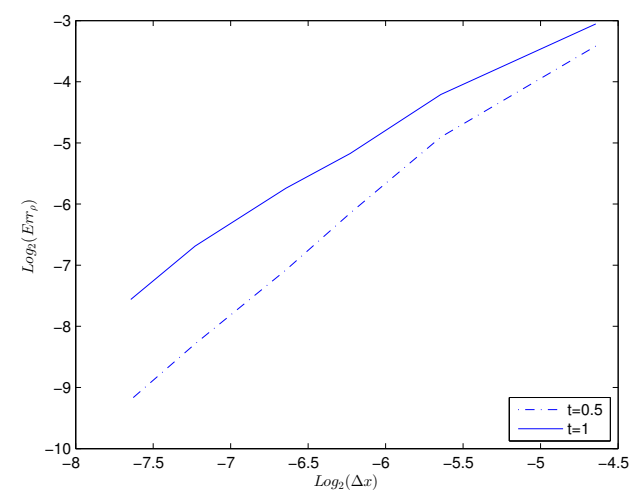

Figure 10: Error curves with respect to spatial step $\Delta x$ for $\rho$ in $\Omega_{T}$ at $t=0.5$ and $t=1$.

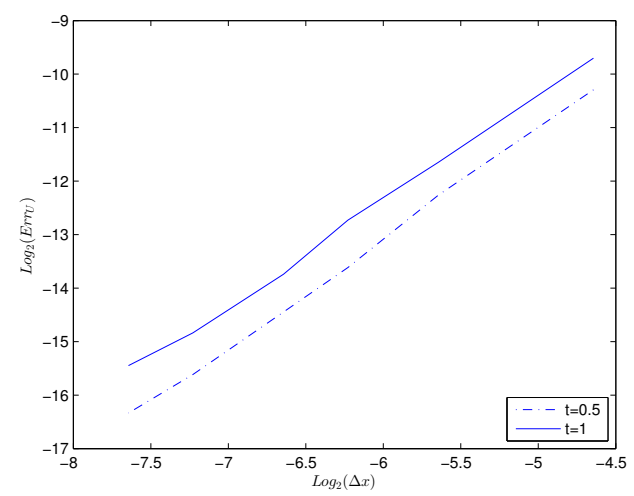

Figure 11: Error curves with respect to spatial step $\Delta x$ for $U$ in $\Omega_{T}$ at $t=0.5$ and $t=1$.

\section{Remark 4.1.}

We notice that the two elliptic equations have relative errors which depend on convergence criteria given in (129) and (130) with $C_{\rho_{e}}=C_{\rho_{T}}=1$ and $N_{T}=N_{e}=2$. These criteria lead to a $10^{-2}$ order relative error for both solutions of elliptic equations.

\subsection{Study of an action potential in heart and torso}

With this second set of data, we aim to reproduce a realistic electrical wave propagation in heart and torso. For that, we apply an external density current within source term $f_{i s}$ in a short time scale $\left(t \in\left[0, T_{a c t}\right]\right.$ ) which represents external applied current (electroshock for example). This applied current involves action potentials (AP) which are generated by the movement of ions into and out of cardiac cells (i.e. biological manifestation of electrical cells excitation) with diffusion in torso region. This potential leads us then to study the evolution of the transmembrane potential $\rho$ and ionic variable $U$. The purpose of the next analysis is to derive the following standard four phases of the action potential (see e.g. [10] and references therein).

- Phase I: Depolarization. In a short time scale (order of $\mathcal{T}_{\text {in }}$ ), transmembrane potential (TMP) changes from its resting state $\rho_{\min }$ to its maximum $\rho_{\max }$ when cells are depolarized. This sudden change induces opening of ionic channels, so ionic variable $U$ leaves its equilibrium state to falling down. 
- Phase II: Excited phase. In a long time scale (order of $\mathcal{T}_{\text {open }}$ ), TMP $\rho$ begins to fall slowly. During this phase, ionic channels are open and ionic variable $U$ goes to its minimum.

- Phase III: Repolarization. In a short time scale (order of $\mathcal{T}_{\text {out }}$ ), TMP goes back towards resting potential of $\rho_{\min }$ to prepare the cells for a new cycle of depolarization. During this phase, ionic channels are gradually inactivated. That induces inversion of ionic variable slope.

- Phase IV: Recovery period. In a long time scale (order of $\mathcal{T}_{\text {close }}$ ), TMP reaches the resting potential $\rho_{\min }$. During this time, ionic variable slowly goes back to its equilibrium state and ionic channels are closed at resting TMP.

To reproduce this behavior, we take the values of the parameters given in Table 1 , we choose discretization parameters $\Delta \mathbf{x}=1 / 50, \Delta t=\Delta \mathrm{x}^{2}$ and convergence criteria parameters $C_{\rho_{e}}=C_{\rho_{T}}=1$ and $N_{e}=N_{T}=3$, we fix $L_{H}=15 \mathrm{~cm}$ and $L_{T}=45 \mathrm{~cm}$, and we fix $K_{i}=0.003 \mathrm{~S} . \mathrm{cm}^{-1}, K_{e}=0.003 \mathrm{~S} . \mathrm{cm}^{-1}$ and $K_{T}=0.009 \mathrm{~S} . \mathrm{cm}^{-1}$, and we choose the initial data as follows:

$$
\rho(\mathbf{x}, 0)=\rho_{\min }, U(\mathbf{x}, 0)=\frac{1}{\left(\rho_{\max }-\rho_{\min }\right)^{2}} .
$$

Moreover, in order to model electrical excitation within the myocardium (due to heart beat or external applied current), we apply a given external stimulus during a short time scale $\left(0<t-\mathcal{T}_{d}<T_{a c t}\right.$, where $\mathcal{T}_{d}$ is a time-delay), and we define $f_{i s}$ as $(\mathbf{x}=(x, y))$

$$
f_{i s}(\mathbf{x}, t)=I_{a p p} \chi_{H}(\mathbf{x}) \chi_{\left[0, T_{a c t}\right]}\left(t-\mathcal{T}_{d}\right) \chi_{\text {prop }}\left(\mathbf{x}, t-\mathcal{T}_{d}\right) \Phi(\mathbf{x}),
$$

where $I_{a p p}$ is the amplitude of the external applied stimulus with $I_{a p p}=10^{4}$, and the functions $\chi_{H}, \chi_{\left[0, T_{a c t}\right]}$ and $\chi_{\text {prop }}$ are defined by

$$
\begin{gathered}
\chi_{H}(\mathbf{x})=\left\{\begin{array}{l}
1 \text { if } \mathbf{x} \in \Omega_{H}, \\
0 \text { else }
\end{array}\right. \\
\chi_{\left[0, T_{a c t}\right]}(t)=\left\{\begin{array}{l}
1 \text { if } t<T_{a c t}, \\
0 \text { else }
\end{array}\right. \\
\chi_{\text {prop }}(\mathbf{x}, t)=\left\{\begin{array}{l}
1 \text { if } x+y<2 t / T_{a c t}, \\
0 \text { else. }
\end{array}\right.
\end{gathered}
$$

The last characteristic is associated to wave propagation as a diagonal which evolves from left bottom corner to right top corner. The function $\Phi$, which corresponds to the shape of the electrical wave, is defined by

$$
\Phi(\mathbf{x})=1-\frac{1}{L_{H}}\left(x-\frac{L_{H}}{2}\right)^{2}-\frac{1}{L_{H}}\left(y-\frac{L_{H}}{2}\right)^{2} .
$$

Figure 12 shows how the transmembrane potential $\rho$ taken in the middle point of domain $\Omega_{H}$ evolves according to the AP (after electrical wave passage), and we recover clearly the four phases. We present on Figure 13 the ionic behavior in this middle point with evolution of ionic variable $U$.

However, we can see these main phases with 3-dimensional representation of $\rho$ and $U$ at different moments. In a first time, at $t=0.3 s$, the AP involves depolarization of the left bottom corner (Figure 14) of $\Omega_{H}$ and ionic variable $U$ begins to fall down (Figure 15). Then, at $t=0.5 \mathrm{~s}$ (Figure 16), the electrical wave has covered more than the half of heart domain and we can see the different phases of AP: the left bottom corner is already on his recovery period (Phase IV), whereas its neighborhood is on the repolarization phase (Phase III) and we observe the plateau inherent to excited phase (Phase II) just behind the depolarized area (Phase I). In term of ionic variable, we also see the four phases with the activation of ionic channels in left bottom corner at $t=0.3 \mathrm{~s}$ (Figure 15). At $t=0.5 \mathrm{~s}$ 


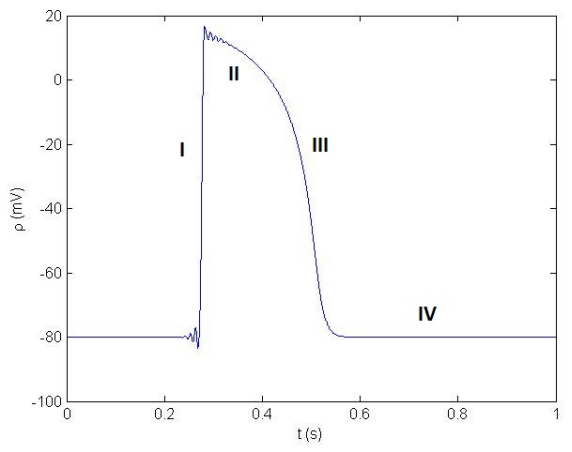

Figure 12: Evolution of transmembrane potential $\rho$ in middle of the heart (with four phases of AP).

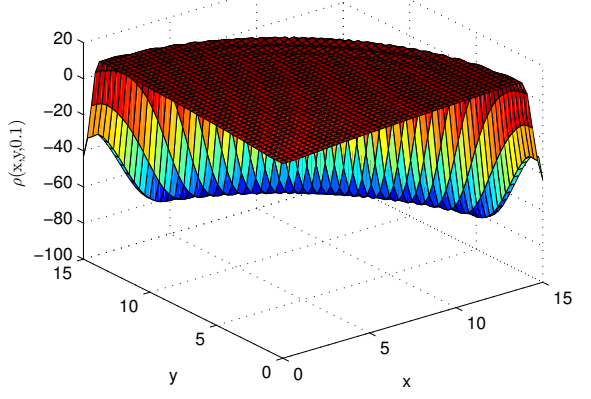

Figure 14: Potential $\rho$ in $\Omega_{H}$ at $t=0.3 s$.

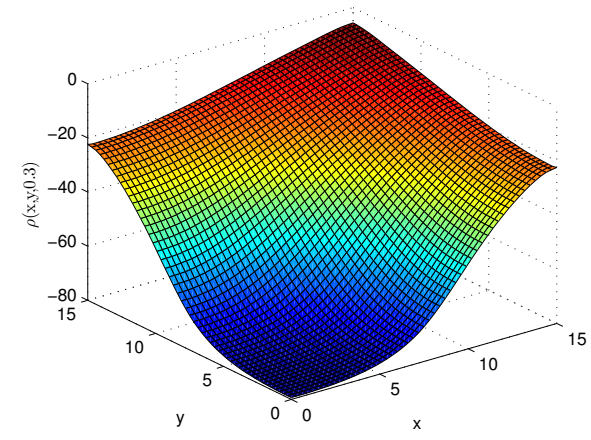

Figure 16: Potential $\rho$ in $\Omega_{H}$ at $t=0.5 s$.

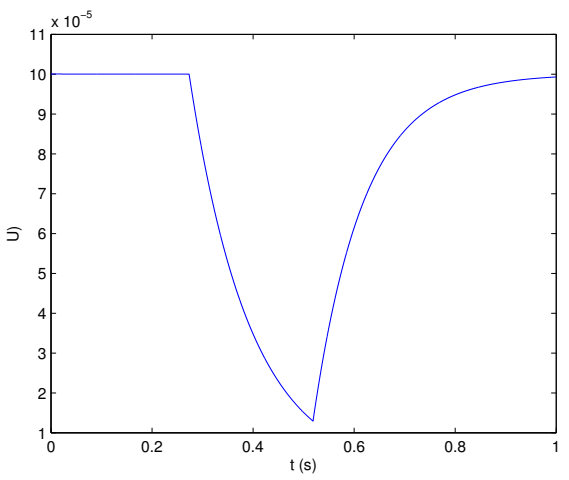

Figure 13: Evolution of ionic variable $U$ in middle of the heart.

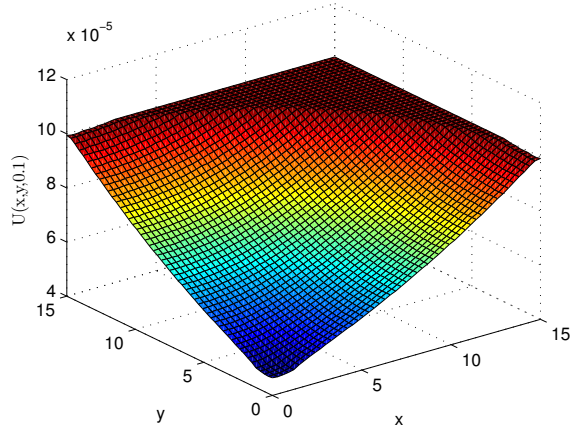

Figure 15: Ionic variable $U$ in $\Omega_{H}$ at $t=$ $0.3 s$

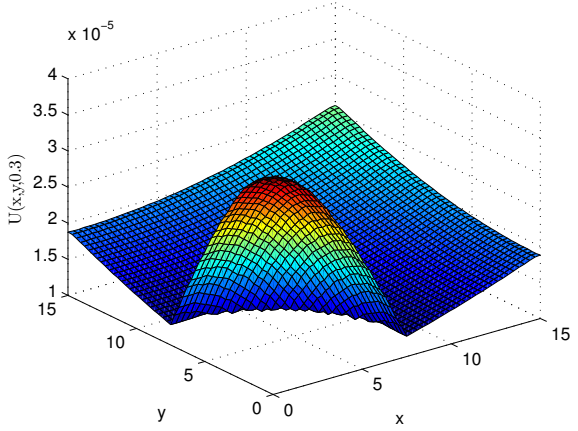

Figure 17: Ionic variable $U$ in $\Omega_{H}$ at $t=$ $0.5 s$ 

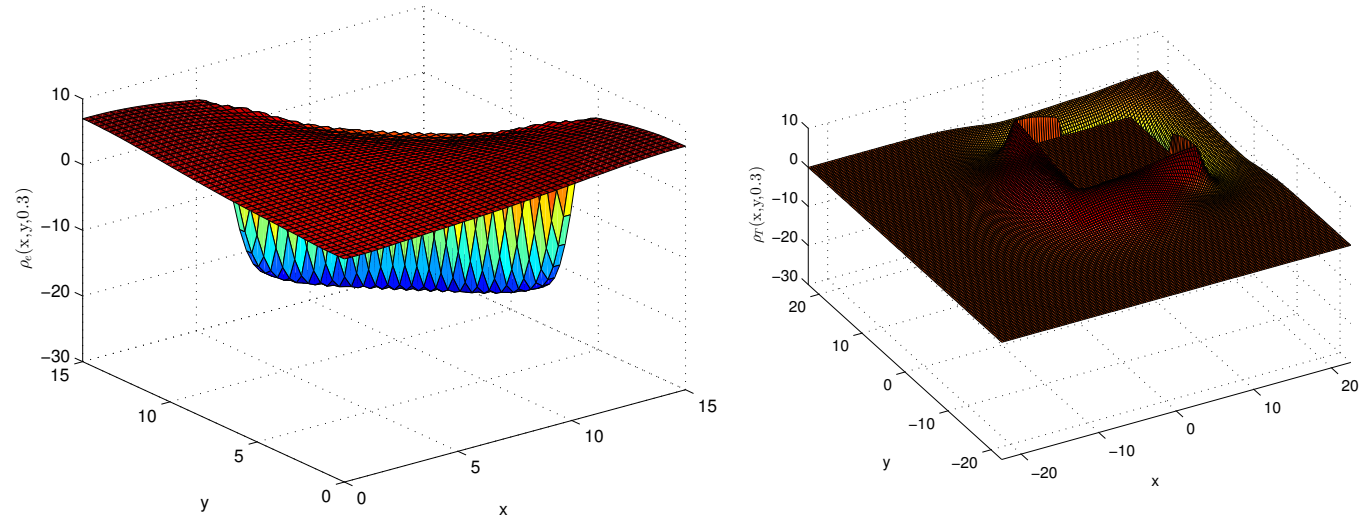

Figure 18: Potential $\rho_{e}$ in $\Omega_{H}$ at $t=0.3 s$.

Figure 19: Potential $\rho_{T}$ in $\Omega_{T}$ at $t=0.3 \mathrm{~s}$
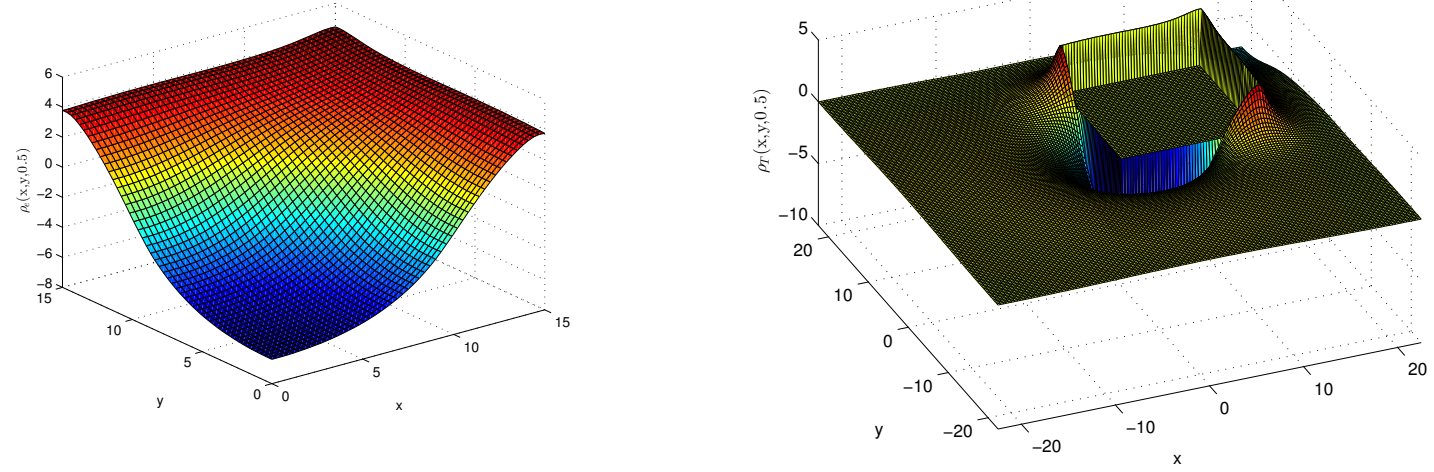

Figure 20: Potential $\rho_{e}$ in $\Omega_{H}$ at $t=0.5 s$.

Figure 21: Potential $\rho_{T}$ in $\Omega_{T}$ at $t=0.5 \mathrm{~s}$ 
(Figure 17), as for transmembrane potential, we can identify the four phases during the propagation of the electrical wave through domain $\Omega_{H}$.

Figures 18 and 20 show that these four phases are visible through the extracellular potential $\rho_{e}$ at respectively $t=0.3 \mathrm{~s}$ and $t=0.5 \mathrm{~s}$. Then, as Figures 24 to 27 show for $\Delta \mathbf{x}=1 / 30$ to $1 / 100$ in middle points of $\Omega_{H}$ and $\Omega_{T}$, there is a few perturbation in the numerical solution, when a sudden change arises, due to chosen stopping criteria. This noise is tending to disappear totally with thinner discretization. Finally, we present on Figures 19 and 21 the diffusion of the electrical wave in the torso at $t=0.3 \mathrm{~s}$ and $t=0.5 \mathrm{~s}$.

To complete the analysis, we study the relationship between the transmembrane potential $\rho$ and ionic variable $U$, and their mutual influence. For this, we introduce the following normalized terms

$$
\rho_{N}=\frac{\rho-\rho_{\min }}{\left|\rho_{\max }-\rho_{\min }\right|}, U_{N}=\frac{U-U_{\min }}{\left|U_{\max }-U_{\min }\right|} .
$$

We can see the correlation between transmembrane potential and ionic movements through the cell membrane in Figure 22. In particular, we clearly see that ionic variable begins to converge slowly to its equilibrium state at the beginning of the recovery period whereas transmembrane potential is already close to its equilibrium.

Finally, in Figure 23 we perform the phase plan curve of $\rho_{N}$ and $U_{N}$. After initialization at $t=0 \mathrm{~ms}$, the point $A$ corresponds to equilibrium state (i.e. $\rho=\rho_{\text {min }}$ and $\left.U=1 /\left(\rho_{\max }-\rho_{\min }\right)^{2}\right)$. We observe that $\rho$ grows to $\rho_{\max }$ in a shorter time scale before the ionic variable $U$ starts to fall down (because $\rho>\rho_{\text {gate }}$ ). This depolarization phase (Phase I) leads us to point $B$. Then, between points $B$ and $C$, we are in the excited phase (Phase II) which numerically corresponds to $\mathcal{I}_{i o n}(\rho, U)=0$. After point $C, \rho$ goes fast to $\rho_{\min }$ while $U$ varies slightly (Phase III). Finally, from point $D$ to the end, $\rho$ is near to its resting state and $U$ goes back to its equilibrium state (Phase IV).

These analyzes led us to verify the efficiency of our coupled modified LBM. Indeed, realistic behavior of the interactions between electrical potentials and ionic movements in heart-torso coupling (which is a critical numerical issue), has been demonstrated by the different simulations. The four phases of the AP are clearly reproduced. Our method remained stable and robust even if the solutions of elliptic equations contain some very local noises due to stopping criteria and sudden changes in dynamics.

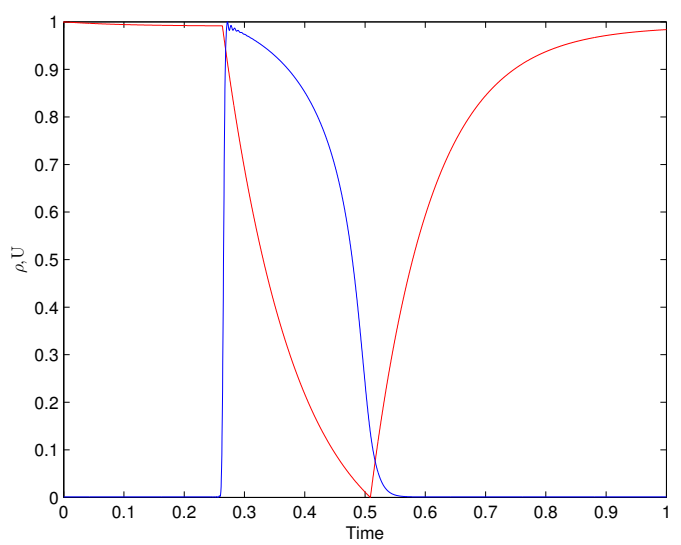

Figure 22: Evolution of $\rho_{N}$ and $U_{N}$ in the middle of heart.

\section{Conclusion and commentary}

An efficient and stable coupled LBM to solve a two-dimensional heart-torso coupled bidomain model is developed. From the Chapman-Enskog expansion analysis, the bidomain 


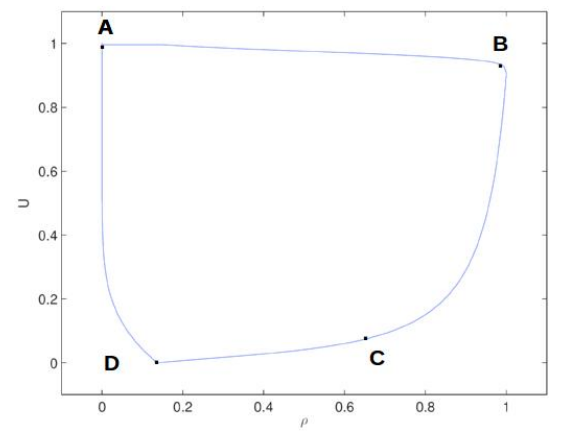

Figure 23: $\left(\rho_{N}, U_{N}\right)$ phase plan portrait.
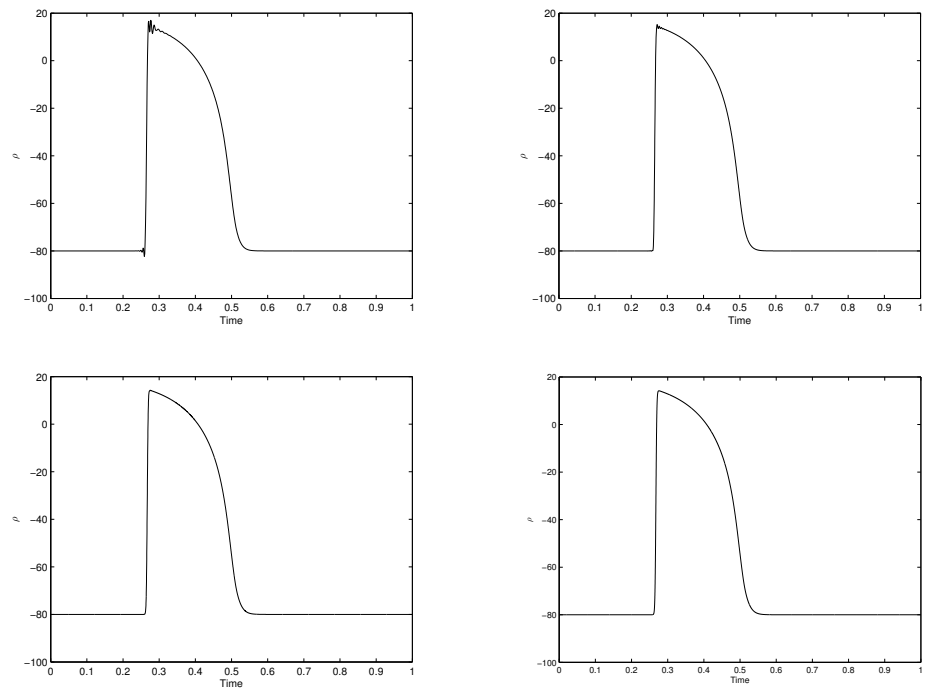

Figure 24: $\rho$ for $\mathbf{x}=(7.5,7.5)$ for $\Delta \mathbf{x}=1 / 30,1 / 50,1 / 75$ and $1 / 100$.

system which is a coupled of reaction-diffusion, elliptic and ODE equations, can be correctly recovered by our modified LBM. This method is easy to implement. The preliminary results presented in this work show the capability of this method to capture the distribution of the electrical wave. It is clear that, due to the multi-scale nature of the system, the Cartesian grid used in our preliminary simulations is not very sufficient to compute in a computationally efficient manner real life clinical situations (see e.g. [17], [32] ) with complex geometry which is in general computationally expensive. Therefore, it is expected to solve the Lattice Boltzmann system on adapted Cartesian or triangular unstructured grids as e.g., in [33, 18] and the references therein. Moreover, in order to overcome the limitations of the constraint CFL stability condition, we extend the method to implicit or semi-implicit time schemes, e.g., by using the $\theta$-method (with $\theta \in[0,1]$ ) or Runge-Kutta methods, coupled with adaptive time stepping strategies, as e.g. in [21] and the references therein. This coupled LBM method will be shown in a forthcoming paper for more general coupled models with realistic complex geometries. It would be interesting to use this developed method with observations coming from experimental data and a more complete description of the biophysical model of electrical cardiac activity. Moreover, since time delays in signal transmission are inevitable and a small delay can affect considerably the resulting electrical activity in heart and thus the cardiac disorders therapeutic treatment, it is then necessary to analyze the impact of delays on the dynamical behaviors of such a system by considering time-delays models with continuous and impulsive treatment (see e.g. [2]). 

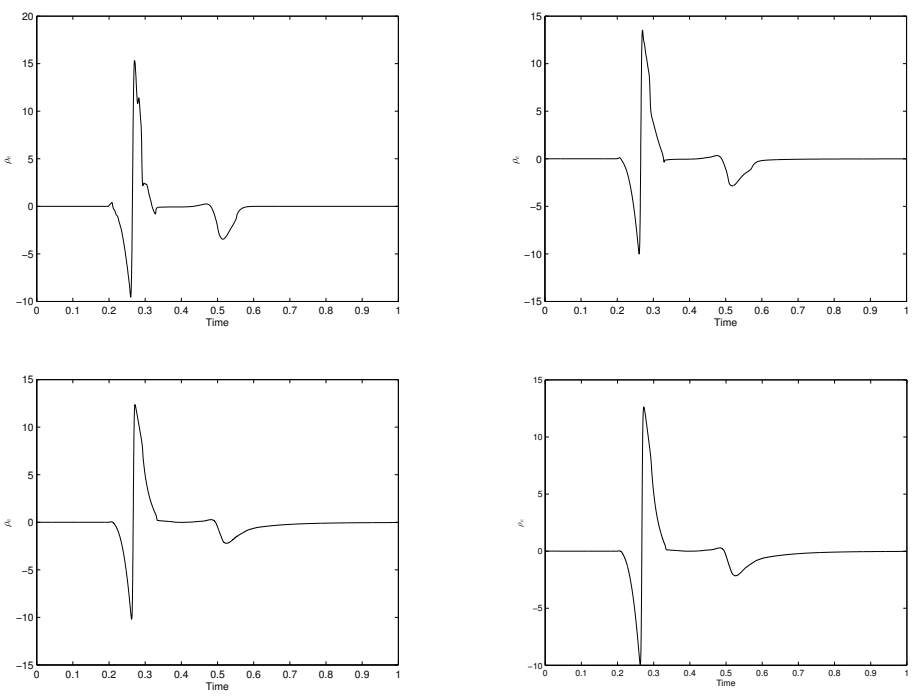

Figure 25: $\rho_{e}$ for $\mathbf{x}=(7.5,7.5)$ for $\Delta \mathbf{x}=1 / 30,1 / 50,1 / 75$ and $1 / 100$.
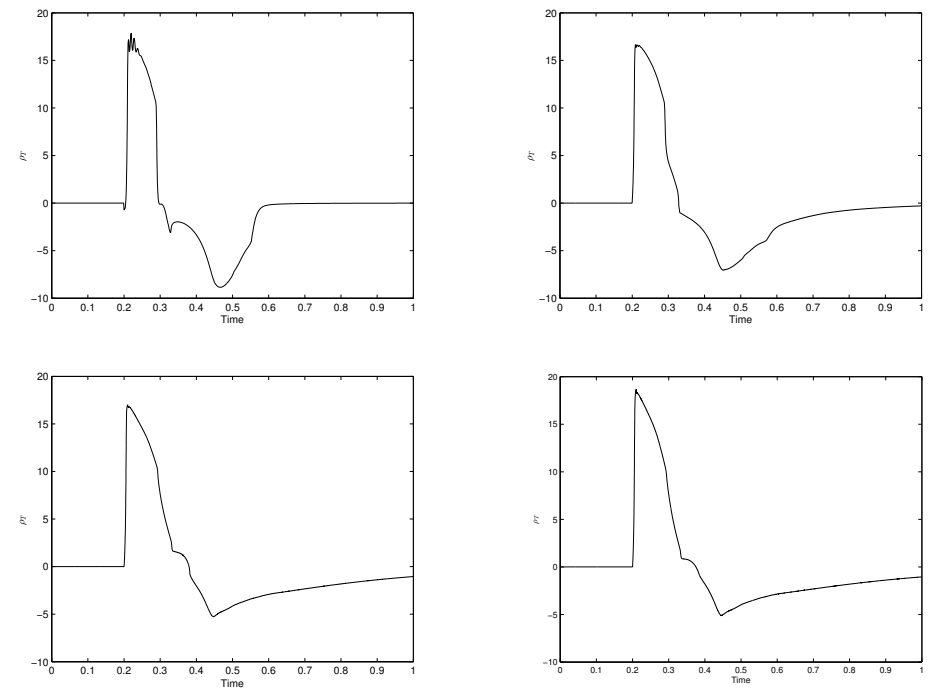

Figure 26: $\rho_{T}$ for $\mathbf{x}=(0,0)$ for $\Delta \mathbf{x}=1 / 30,1 / 50,1 / 75$ and $1 / 100$

In order to get even closer to a more realistic calculation, it is necessary to study, in the future, this method coupled with optimization technique and, optimal and robust control problems by using the approach developed in [1, 4].

Acknowledgments: The autors would like to thanks all the referees for their relevant questions, suggestions and comments which lead to improve this paper. 

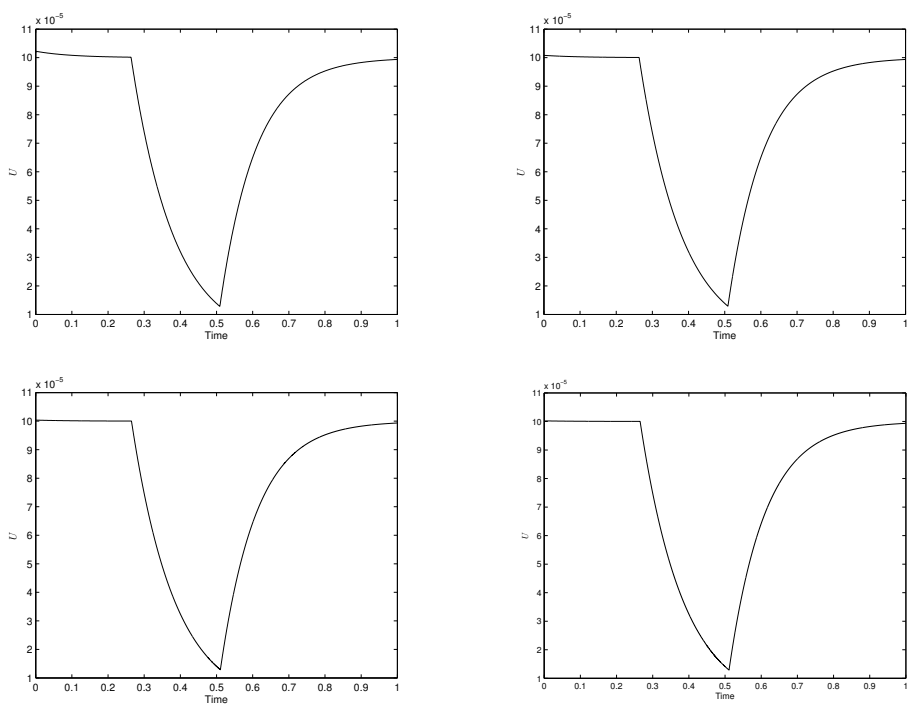

Figure 27: $U$ for $\mathbf{x}=(7.5,7.5)$ for $\Delta \mathbf{x}=1 / 30,1 / 50,1 / 75$ and $1 / 100$

\section{Appendix}

\subsection{Nondimensional model}

We have nondimensionalized (4)-(6) by introducing the following dimensionless quantities (dimensionless variables, parameters and physical domains are denoted with " ")

$$
\begin{aligned}
& \tilde{\mathbf{x}}=\frac{\mathbf{x}}{\bar{L}}, \quad \tilde{t}=\frac{t}{\bar{T}}, \quad \tilde{\nabla}=\bar{L} \nabla, \quad \operatorname{div}=\bar{L} \operatorname{div}, \quad \partial / \partial \tilde{t}=\bar{T} \partial / \partial t, \\
& \mathcal{K}_{e c}=\bar{\sigma} \tilde{\mathcal{K}}_{e c}, \quad \mathcal{K}_{i c}=\bar{\sigma} \tilde{\mathcal{K}}_{i c}, \quad \mathcal{K}_{T}=\bar{\sigma} \tilde{\mathcal{K}}_{T},
\end{aligned}
$$

where $\bar{L}$ is a characteristic length of physical domain $\Omega, \bar{T}$ is a characteristic time and $\bar{\sigma}$ is a characteristic conductivity. The dimensional transmembrane and extracellular potentials $\rho$ and $\rho_{e}$, and thorax potential $\rho_{T}$ are rescaled using

$$
\rho=\bar{\rho} \tilde{\rho}+\rho_{\text {min }}, \quad \rho_{e}=\bar{\rho} \tilde{\rho}_{e}+\rho_{\text {min }}, \quad \rho_{T}=\bar{\rho} \tilde{\rho}_{T}+\rho_{\text {min }}
$$

with $\bar{\rho}=\rho_{\max }-\rho_{\min }$ the characteristic action potential amplitude. The electrophysiological ionic state variable $U$ is rescaled using

$$
\tilde{U}=\bar{\rho}^{2} U \text {. }
$$

Using these adimensional relations, we get finally the dimensionless system

$$
\begin{aligned}
& \frac{\partial \tilde{\rho}}{\partial \tilde{t}}+\tilde{\mathcal{I}}_{i o n}(. ; \tilde{\rho}, \tilde{U})-R_{h} \operatorname{div}\left(\tilde{\mathcal{K}}_{i c} \tilde{\nabla} \tilde{\rho}\right)=R_{h} \tilde{d i v}\left(\tilde{\mathcal{K}}_{i c} \tilde{\nabla} \tilde{\rho}_{e}\right)+\tilde{f}_{i s}, \text { on } \tilde{\mathcal{Q}}_{H} \\
& -R_{h} \tilde{d i v}\left(\left(\tilde{\mathcal{K}}_{e c}+\tilde{\mathcal{K}}_{i c}\right) \tilde{\nabla} \tilde{\rho}_{e}\right)=R_{h} \tilde{d i v}\left(\tilde{\mathcal{K}}_{i c} \tilde{\nabla} \tilde{\rho}\right)+\left(\tilde{f}_{e s}+\tilde{f}_{i s}\right), \text { on } \tilde{\mathcal{Q}}_{H} \\
& \frac{\partial \tilde{U}}{\partial \tilde{t}}=\tilde{H}_{i o n}(. ; \tilde{\rho}, \tilde{U}), \text { on } \tilde{\mathcal{Q}}_{H}
\end{aligned}
$$

and

where

$$
-R_{h} \tilde{\operatorname{div}}\left(\tilde{\mathcal{K}}_{T} \tilde{\nabla} \tilde{\rho}_{T}\right)=\tilde{f}_{T}, \text { on } \quad \tilde{\mathcal{Q}}_{T}
$$

$$
\begin{aligned}
& R_{h}=\frac{\bar{\sigma} \bar{T}}{\bar{L}^{2} \kappa c_{m}} \\
& \tilde{f}_{i s}=\frac{\bar{T}}{c_{m} \bar{\rho}} f_{i s}, \quad \tilde{f}_{e s}=\frac{\bar{T}}{c_{m} \bar{\rho}} f_{e s}, \quad \tilde{f}_{T}=\frac{\bar{T}}{\kappa c_{m} \bar{\rho}} f_{T} \\
& \tilde{\mathcal{I}}_{i o n}(\tilde{\mathbf{x}}, \tilde{t} ; \tilde{\rho}, \tilde{U})=\frac{\bar{T}}{c_{m} \bar{\rho}} \mathcal{I}_{i o n}(\mathbf{x}, t ; \rho, U), \\
& \tilde{H}_{i o n}(\tilde{\mathbf{x}}, \tilde{t} ; \tilde{\rho}, \tilde{U})=\frac{\bar{T} \bar{\rho}{ }^{2}}{} H_{i o n}(\mathbf{x}, t ; \rho, U) .
\end{aligned}
$$


Remark 6.1.

If we take for example $\bar{\sigma}=\frac{\bar{L}^{2} \kappa c_{m}}{\bar{T}}, \bar{T}=T$ and $\bar{L}=L_{H}$ then $R_{h}=1, \tilde{\mathcal{Q}}_{H}=[0,1] \times[0,1] \times$ $[0,1]$ and $\tilde{\mathcal{Q}}=\tilde{\Omega} \times[0,1]$, where $\tilde{\Omega}=\left[-\frac{L_{T}}{L_{H}}, \frac{L_{T}}{L_{H}}\right] \times\left[-\frac{L_{T}}{L_{H}}, \frac{L_{T}}{L_{H}}\right]$.

\subsection{Implementation and Algorithm}

Our goal is to develop an efficient LBM for bidomain system. In this work, in order to validate the method, we just focus on stability and accuracy of the method for coarse discretization without any specific optimization. So we make our simulation in a beta version of our program, and the characteristic execution time can be significantly reduce with using some Matlab functions or parallelizations. We want to control all the stages of build, and we need to represent every functions at each stage. That is why some stages are not optimized yet, even if we can do it easily (and so reduce the CPU time). The other main reason is that some stages are already implemented for complex boundary and complex domain for further works; the initialization stage or boundary treatment could be simplified for simpler boundaries. By the same way, we introduce some functions in order to have easy graphical representations. For all these reasons, we insist on the non optimal nature of our present code, and the characteristic CPU times we obtain are not revealing of the efficiency of LBM.

Moreover, the way we suggested for parallelization has not already been studied further by ourselves. Indeed, the two-stage build of the method guarantees that we can compute all local stages of the method with dividing the mesh between different threads. That includes local summations to recompose all the macroscopic values, the local definition of the equilibrium functions, second terms and link functions, and the local treatment of collision stage. Then, the streaming process can be resume as an index substitution between distribution function of adjacent nodes witch can also be separate for each velocity $\mathbf{e}_{i}$. This proposed parallelization will be done in a future work.

All the steps of this algorithm are represented in Figure 28. The step $S 1$ is the initialization of the mesh (including vectors $\mathbf{e}_{i}$, vectors of boundaries index, or usual objects for graphical representations), the non-dimentionalization on time and space according to system (142), and definition of initial conditions. The step $S 2$ is the treatment of the parabolic equation and of the ODE of system (142), and the both subroutines $S 3$ and $S 4$ correspond to the treatment of the elliptic equation of the system (142) in the heart and elliptic equation (143) in the thorax.

To estimate typical computionnal times, we have made our simulation with Matlab $2016 \mathrm{~b}$ and with Intel core i5 processors. We present in Table 3 the different typical computational times for each part $S 1$ to $S 4$ of the algorithm in $\Omega \times(0, T)$ with $T=1$ according to scale $\Delta \mathbf{x}=1 / 50, \ldots, 1 / 100, \Delta t=\Delta \mathbf{x}^{2}$, and convergence criteria parameters $C_{\rho_{e}}=C_{\rho_{T}}=1$ and $N_{e}=N_{T}=3$. The execution time of both elliptic equations treatments are strongly related to the convergence criteria. With this simple implementation without optimization or parallelization, the execution time grows quickly according to the number of iterations and to the number of nodes. The first scaling $\Delta x=1 / 50$ induces 2500 iterations to reach $T=1$ and each domain contains 2601 nodes, and the last one $\Delta x=1 / 100$ induces $10^{4}$ iterations for 10201 nodes for each domain. So we can conclude that execution time for each iteration is proportional to the number of nodes.

\begin{tabular}{ccccc}
\hline$\Delta x$ & $\mathrm{~S} 1$ & $\mathrm{~S} 2$ & $\mathrm{~S} 3$ & $\mathrm{~S} 4$ \\
$1 / 50$ & 1.0598 & 58.9528 & 71.9957 & 217.8366 \\
$1 / 75$ & 7.8410 & 201.9650 & 243.5739 & 657.5601 \\
$1 / 100$ & 22.5423 & 764.5007 & 1042.5311 & 2567.0278 \\
\hline
\end{tabular}

Table 3: Typical computational time (in second) for each part of the algorithm in $\Omega \times(0,1)$. 


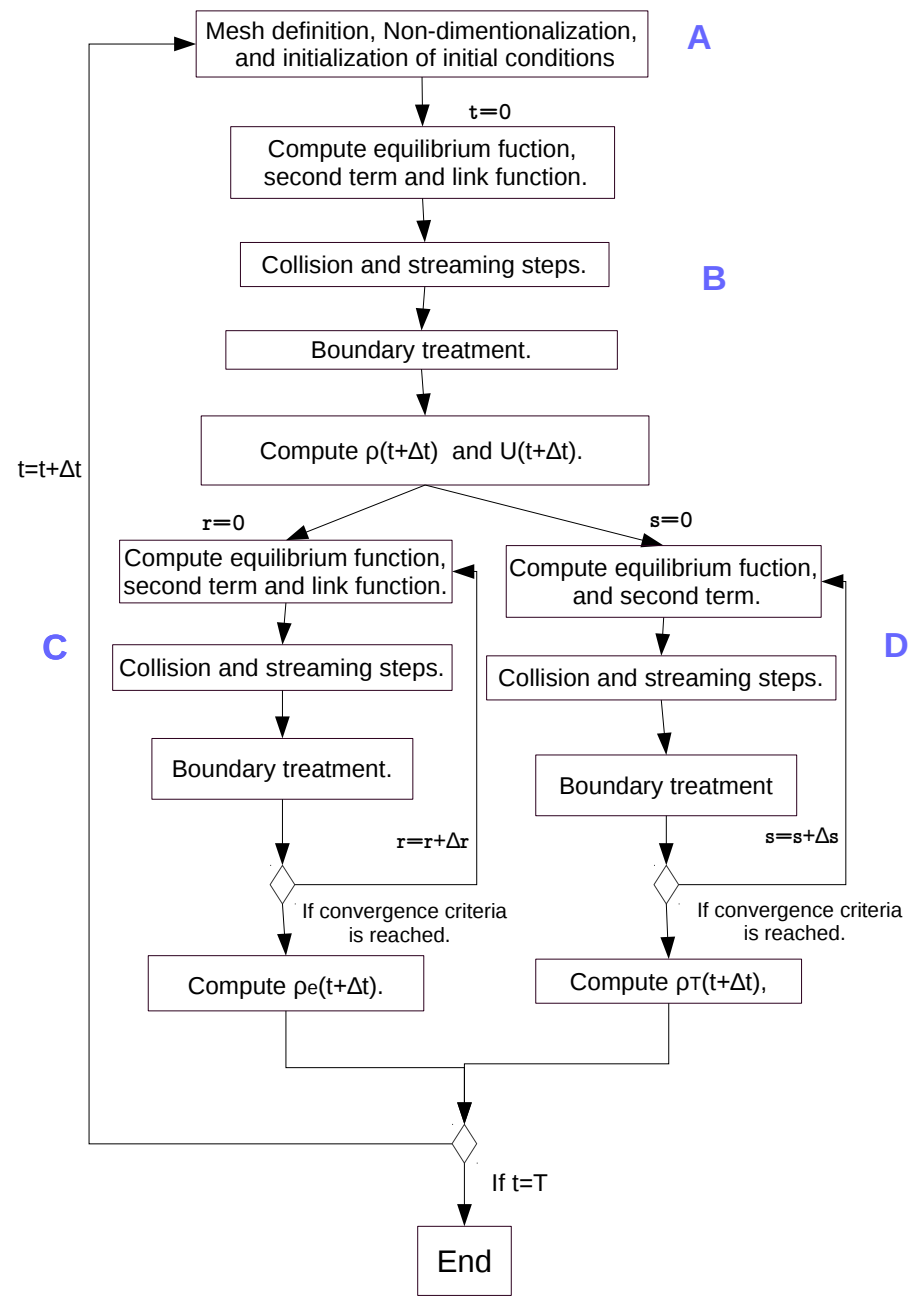

Figure 28: Step by step representation of the algorithm.

\section{References}

[1] A. Belmiloudi, Mathematical modeling and optimal control problems in brain tumor-targeted drug delivery strategies, International Journal of BiomathEMATICS, 60 PAGES, TO APPEAR.

[2] A. Belmiloudi, Dynamical behavior of nonlinear impulsive abstract partial differential equations on networks with multiple time-varying delays and mixed boundary conditions involving time-varying delays, J. Dynam. Control Systems, 21, (2015), 95-146.

[3] Belmiloudi A., Robust control problem of uncertain bidomain models in cardiac electrophisiology, J. Coupled Systems \& Multiscale Dynamics, 19, 332-350, 2013.

[4] Belmiloudi A., Stabilization, optimal and robust control. Theory and applications in biological and physical sciences, SPRINGER-VERLAG, LONDON, (2008).

[5] Bhatnagar P.L., Gross E.P., Krook M., A model for Collision Processes in Gases Small Amplitude Processes in Charged and Neutral One-Component Systems, Phys. Rev., 94 (1954), Pp. 511-525. 
[6] Boltzmann L., Weitere Studien uber das Warme gleichgenicht unfer Gasmolakuler, Sitzungsberichte Akad. Wiss., 66, 275, 1872.

[7] Boulakia M., Cazeau S., Fernández M. A., Gerbeau J.-F., Zemzemi N., Mathematical Modeling of Electrocardiograms: A Numerical Study, AnNAls of Biomedical EngineERing, 38, PP 1071-1097, 2009.

[8] Boulakia M., Fernandez M. A., Gerbeau J.-F., Zemzemi N., A Coupled System of PDEs and ODEs arising in Electrocardiograms Modelling. RESEARCH REPORT, RR-6352, INRIA. 2007 PP.24.

[9] Bourgault Y., Coudière Y., Pierre C., Existence and uniqueness of the solution for the bidomain model used in cardiac electrophysiology, NONLINEAR ANALREAL, 10 458-482, 2009.

[10] Britton N. F. , Essential Mathematical Biology. Springer Undergrad. Math. SeRIES (2003).

[11] J.O. Campos, R.S. Oliveir, , R.W. dos Santos, B.M. Rocha., Lattice Boltzmann method for parallel simulations of cardiac electrophysiology using GPUs, J. Comput. Appl. Math., 295, 70-82, 2016.

[12] Chai Z., Shi BC., Novel Boltzmann model for the Poisson equation, Applied Mathematical Modeling, 32, 2050-2058, 2008.

[13] Chapman S., Cowling T.G., The mathematical theory of non-uniform gases, Cambridge University Press, England, (1990).

[14] Clayton R.H., Bernus O, Cherry EM, Dierckx H, Fenton FH, Mirabella L, Panfilov AV, Sachse FB, Seemann G, Zhang H., Models of cardiac tissue electrophysiology: progress, challenges and open questions, PROGRESS IN BIOPHYSics AND Molecular Biology 104 (2011), 22-48.

[15] Corre S., Belmiloudi A., Coupled Lattice Boltzmann Modeling of Bidomain type models in Cardiac Electrophysiology. In Mathematical and Computational Approaches in Advancing Modern Science and Engineering, eds. J. Bélair, I. Frigaard, H. Kunze, R., Melnik, J. Spiteri, 209-221, SpringerVerlag, Switzerland, 2016.

[16] Djabella K., Landau M., Sorine M., A two-variable model of cardiac action potential with controlled pacemaker activity and ionic current interpretation. 46 $\mathrm{TH}$ IEeE Conf. Decis. Control (2007), New Orleans, LA, USA, 5186-5191.

[17] Ellenbogen K. A., Wilkoff B. L., Kay G. N., Lau C. P.Clinical Cardiac Pacing, Defibrillation and Resynchronization Therapy. Elsevier Health ScIENCEs,Philadelphia, (2006).

[18] Fan Z., Zhao Y., Kaufman A., He Y., Adapted Unstructured LBM for Flow Simulation on Curved Surfaces, IN ACM SIGGRAPH, PP 245-254, 2005.

[19] Fenton F., Karma A., Vortex dynamics in three-dimensional continuous myocardium with fiber rotation: Filament instability and fibrillation., CHAOS 8 (1998) $20-47$.

[20] Zheng C.-G., Shi B., Non-equilibrium extrapolation method for velocity and pressure boundary conditions in the lattice Boltzmann method, Chinese Physics, 4 , $366,2002$.

[21] Huang J., Yang C., Cai X.-C., A Fully Implicit Method for Lattice Boltzmann Equations, SiAM J. Sci. Comput., 37(5), Special Section, S291-S313, 2015.

[22] Huang K., Statistical Mechanics, John Wiley \& Sons, Inc., Canada, 1963. 
[23] Ladd A.J.C., Verberg, R., Lattice-Boltzmann Simulations of Particle-Fluid Suspensions, Journal of Statistical Physics, 104, 1191-1251,2001.

[24] Lines G.T., Grottum P., Tveito A., Modeling the electrical activity of the heart: A Bidomain Model of the ventricles embedded in a torso, Comput VISUAL SCI (2003) 5, 195.

[25] LuO C.H., Rudy Y., A dynamic model of the cardiac ventricular action potential: I. simulations of ionic currents and concentration changes. CIRC. REs.74 (1994) 1071-1096.

[26] Mitchell C.C., Schaeffer D.G., A two-current model for the dinamics of cardiac membrane. Bull. Math. Biol., 65:767-793, 2003.

[27] Fitzhugh R., Impulses and physiological states in theorical models of nerve membrane. BIOphys. J. 1:445-465, 1961.

[28] Premnath K.N., Abraham J., Three-dimensional multi-relaxation time (MRT) lattice-Boltzmann models for multiphase flow, Journal OF COMPUTATIONAL PhYSICS, VOL. 224,PP 539-559, 2007.

[29] Rioux M., Burgault Y., A Predictive Method Allowing the use of a Single Ionic Model in Numerical Cardiac Electrophysiology, ESAIM: M2AN 47, PP 987-1016, 2013.

[30] N. Satofuka, T. Nishioka, Parallelization of lattice Boltzmann method for incompressible flow computations, Computational Mechanics Vol. 23, Number 2, PAGE 164, 1999.

[31] Sharomi O., Spiteri R., Convergence order vs. parallelism in the numerical simulation of the bidomain equations, Journal of Physics: Conference Series, $385,1-6,2012$.

[32] Schulze, W. H.ECG Imaging of Ventricular Activity in Clinical Applications, Vol. 22, KIT Scientific Publishing (2015).

[33] Valero-Lara P., Jansson J., A Non-uniform Staggered Cartesian Grid Approach for Lattice-Boltzmann Method, Procedia Computer Science, 51, 296$305,2015$.

[34] Xiang X., Wang Z., Shi B., modified lattice boltzman scheme for nonlinear convection diffusion equations, Commun Nonlinear Sci Numer Simulat, 17, 24152425, 2012.

[35] Yang X., Shi B., Chai Z., Coupled lattice Boltzmann method for generalized Keller-Segel chemotaxis model, Comput. Math. ApPl., 12, 1653-1670, 2014.

[36] Yin X., Zhang J. An improved bounce-back scheme for complex boundary conditions in lattice Boltzmann method, Journal of Computational Physics, 231, 4295-4303, 2012.

[37] Chai Z., Guo Z., Lu J., Shi B., Zhang T., General bounce-back scheme for concentration boundary condition in the lattice-Boltzmann method, AMERICAN PHYSICal Society, Vol. 85, 2012.

[38] Zhi S., Ming C., And Jing L., Parallelization of Lattice Boltzmann method using MPI domain decomposition technology for a drop impact on a wetted solid wall, Int. J. Model. Simul. Sci. Comput.,Vol. 05, 2014. 